\title{
ENSAYES EN MESA VIBRADORA DE EDIFICIOS MINIATURA CON MUROS ESTRUCTURALES DE CONCRETO CONVENCIONALES Y AUTOCENTRADOS
}

\author{
Rafael Salinas-Basualdo ${ }^{(1)}$, Mario E. Rodríguez ${ }^{(2)}$ y Roque A. Sánchez ${ }^{(3)}$
}

\begin{abstract}
RESUMEN
Este trabajo describe los resultados de ensayes en mesa vibradora de tres especímenes miniatura de cinco pisos con sistemas muro-marco, diseñados por sismo con criterios semejantes a los empleados para edificios con sistemas duales convencionales. Un espécimen tuvo un muro estructural convencional y dos especímenes tuvieron muros estructurales autocentrados. Los muros autocentrados fueron de concreto presforzado con tendones de postensado no adherido, y barras de refuerzo de acero ASTM A615 entre la base del muro y la cimentación, diseñadas como elementos disipadores de energía. Se desarrollaron modelos de análisis estructural que fueron calibrados para reproducir los resultados experimentales de los especímenes ensayados, obteniéndose de estos análisis resultados comparables a los obtenidos en las mediciones experimentales. Los ensayos mostraron que los especímenes muro-marco con muros autocentrados presentaron en general un desempeño semejante al observado en el espécimen muro-marco con muro convencional. Una mayor participación de los muros autocentrados en la respuesta total llevó a un incremento en la sobrerresistencia y una reducción tanto de las distorsiones residuales en la estructura como de las deformaciones residuales en las secciones críticas de los elementos de los marcos.
\end{abstract}

Palabras clave: concreto reforzado; muros estructurales; muros autocentrados; ensayos en mesa vibradora; análisis dinámico no lineal; diseño sismo-resistente

\begin{abstract}
This paper describes results of shaking table tests of three five-story miniature buildings with wallframe systems, designed with seismic design criteria similar to those used in conventional wallframe dual systems. One of the specimens had one conventional structural wall and the other two had self-centering structural walls. Self-centering walls were built with unbonded post-tensioned prestressed concrete and energy dissipators of ASTM A615 steel between the base and the wall foundation. Analytical models were developed for structural analysis, which were calibrated to reproduce the experimental results. Predicted response using the analytical models was comparable to measured response. The tests showed that the specimens with self-centering wall-frame dual systems had in general a behavior similar to that observed in the specimen with a conventional wallframe system. In addition, it was observed that an increase in the participation of the self-centering structural walls in the total seismic response led to an increase in overstrength and to a decrease in
\end{abstract}

Artículo recibido el 21 de diciembre de 2012 y aprobado para su publicación el 19 de agosto de 2013. Se aceptarán comentarios y/o discusiones hasta cinco meses después de su publicación.

(1) Facultad de Ingeniería Civil, Universidad Nacional de Ingeniería, UNI. Lima, Perú; rsalinas@ uni.edu.pe.

(2) Instituto de Ingeniería, Universidad Nacional Autónoma de México, UNAM. México, D.F.; mrod@unam.mx.

(3) Facultad de Ingeniería Civil, Universidad Nacional de Ingeniería, UNI. Lima, Perú; rsanchezm@uni.edu.pe 
residual drifts of the structure as well as in residual deformations in critical sections of the frame elements.

Keywords: reinforced concrete; structural walls; self-centering walls; shaking table test; non-linear dynamic analysis; earthquake-resistant design

\section{INTRODUCCIÓN}

El desarrollo actual de los procedimientos constructivos y de diseño sísmico está orientado a reducir el daño en las estructuras y en el equipamiento de los edificios, considerando los diversos niveles de solicitación sísmica a que están sometidos. En las estrategias innovadoras propuestas para la reducción del daño en las estructuras se emplean, por ejemplo, elementos estructurales prefabricados de concreto con muros autocentrados. La idea para emplear elementos autocentrados en edificios fue estudiada inicialmente por Priestley y Tao (1993) y Stone et al. (1995). Estudios experimentales y analíticos posteriores (Priestley, 1996; Rahman y Restrepo, 2001; Holden et al., 2003; Perez et al., 2007; Restrepo y Rahman, 2007) ampliaron la idea inicial para dar forma actual a la alternativa estructural de los muros autocentrados con postensado sin adherencia. En la actualidad, se cuentan con expresiones para definir algunos aspectos del comportamiento de muros autocentrados, calibradas con resultados de ensayes en muros aislados sometidos a cargas cíclicas, y recomendaciones para el diseño de muros prefabricados con postensado no adherido (ACI-ITG, 2009).

Un muro autocentrado consiste en un muro de concreto reforzado ligado a la cimentación mediante un tendón postensado anclado a la zapata, sin unión monolítica entre el muro y la cimentación. Para dotar al muro de capacidad de disipación de energía ante acciones sísmicas se incluyen barras de acero dúctil, para formar lo que se denomina un sistema híbrido (figura 1). Para una mayor efectividad del preesfuerzo, el tendón no está adherido al muro. Los estudios iniciales que se encuentran en la literatura estuvieron orientados a definir las características elásticas e inelásticas de la relación fuerza-desplazamiento lateral de los muros autocentrados como elementos estructurales aislados.
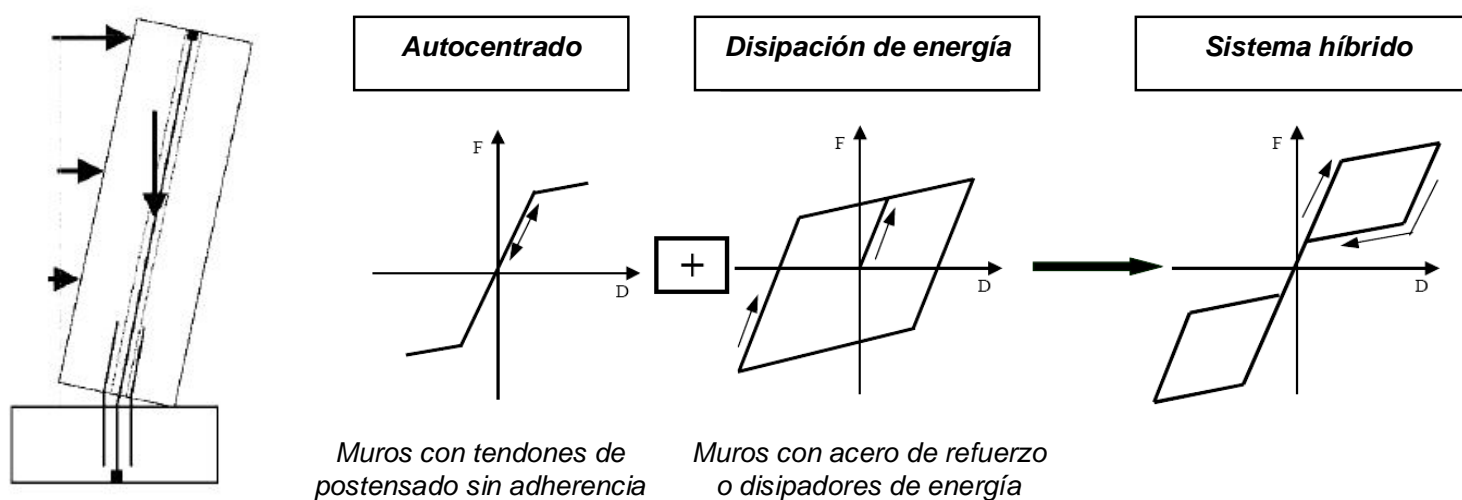

Muros con tendones de
postensado sin adherencia con acero de refuerzo
o disipadores de energía

Figura 1. Muro autocentrado y comportamiento histerético idealizado del sistema híbrido.

(Restrepo y Rahman, 2007; Pampanin, 2005)

Desde la década anterior se ha estudiado el comportamiento de muros de concreto prefabricado con tendones de postensado no adherido (Kurama et al., 1999; Holden et al., 2003; Restrepo y Rahman, 2007). Holden et al. (2003) ensayaron muros a escala 1:2 con cargas laterales cíclicas reversibles y compararon su comportamiento con el de muros de concreto reforzado. Estas investigaciones mostraron las 
características de autocentrado y disminución de los desplazamientos residuales del sistema con postensado no adherido. Restrepo y Rahman (2007) ensayaron muros a escala 1:2 con cargas laterales cíclicas reversibles y compararon el comportamiento registrado en especímenes con postensado no adherido y diferentes proporciones de barras disipadoras de acero dúctil para proveer de disipación de energía; asimismo, propusieron recomendaciones para el diseño de muros no acoplados.

Los sistemas autocentrados pueden ser modelados usando técnicas aparentemente detalladas tales como el uso de elementos finitos o elementos tipo fibra. Sin embargo, una técnica bastante práctica de modelado es el uso de un modelo de plasticidad concentrada, para lo cual generalmente se emplea uno o más resortes rotacionales. El modelo de histéresis puede ser calibrado mediante el uso de resultados experimentales o de una estrategia analítica (Pampanin et al., 2001; Pennucci et al., 2009). Aunque generalmente se considera que el comportamiento histerético de estos elementos puede aproximarse a uno del tipo bandera (figura 1), los trabajos anteriormente citados sobre ensayos de muros con postensado no adherido y barras disipadoras en la base del muro, mostraron que realmente ocurre una reducción progresiva de la rigidez inicial debido a la abertura en la base del muro y el comportamiento no lineal del concreto en la base.

Este estudio hace énfasis en el comportamiento sismorresistente de edificios con sistemas del tipo dual (combinación muro-marco). Para este fin se ensayaron en mesa vibradora tres edificios miniatura que representaban a un sistema estructural sismorresistente del tipo muro-marco (sistema dual), un edificio tenía un muro convencional y los otros dos edificios tenían muros autocentrados. No existe información en la literatura sobre el comportamiento dinámico de edificios con muros autocentrados, y este trabajo pretende contribuir en este aspecto. Además, es relevante comparar el comportamiento de este tipo de sistema dual con uno del tipo convencional, es decir con muros estructurales convencionales colados en sitio.

\section{PROGRAMA EXPERIMENTAL}

Se ensayaron tres especímenes que consistieron en edificios miniatura estructurados con base en marcos de acero y muros de concreto (sistema dual). El espécimen E1 tuvo un muro estructural empotrado en la base, diseñado en forma convencional, y los especímenes A1 y A2 tuvieron muros estructurales autocentrados. Los marcos y los muros se unieron mediante elementos rígidos de acero, en cada nivel, con el fin de transmitir las fuerzas inerciales. Para representar la masa del edificio en cada nivel se emplearon lingotes de acero sobre perfiles metálicos, unidos en su parte superior mediante una placa de acero para formar un diafragma rígido (Rodríguez et al., 2006). Los marcos longitudinales (paralelos a la dirección del ensaye) tuvieron piezas de acero reemplazables, denominadas fusibles, ubicadas en los extremos de las vigas y en la base de las columnas, en las zonas de posible formación de rótulas plásticas (figura 2). Este marco de acero con fusibles fue empleado por primera vez por Kao (Kao, 1998; Rodriguez et al., 2006) en la Universidad de Canterbury, Nueva Zelanda, y marcos con características semejantes han sido empleado en la UNAM, México, en trabajos experimentales llevados a cabo por Blandón (2006) y Sánchez (2008). El diseño de los elementos del marco está concentrado en los fusibles, ubicados en los extremos de las vigas de todos los pisos y en la base de las columnas del primer piso, y el objetivo de este diseño es que estos fusibles tengan la rigidez, la resistencia y la capacidad de disipación de energía necesarias para que los muros alcancen el nivel de comportamiento inelástico que se pretende estudiar. El muro convencional del espécimen E1 fue de concreto reforzado, el diseño del refuerzo se realizó empleando las Normas Técnicas Complementarias para Diseño de Estructuras de Concreto del Distrito Federal (Gaceta Oficial del Distrito Federal, NTCC, 2004). Los muros autocentrados de los especímenes A1 y A2 fueron de concreto reforzado, con cables de postensado no adheridos y barras disipadoras de acero en la base. Las 
dimensiones de estos especímenes y su diseño se basó en las propuestas de Restrepo y Rahman (2007) y en el documento ACI-ITG-5.2-09 (ACI-ITG, 2009).

Para el diseño de los muros se empleó un concreto con resistencia especificada a la compresión, $f_{c}^{\prime}$, igual a $34.3 \mathrm{MPa}$ y un esfuerzo especificado de fluencia, $f_{y}$, igual a $412 \mathrm{MPa}$ para el acero de refuerzo del muro y las barras disipadoras, el cual fue del tipo ASTM A615. Los fusibles del marco se diseñaron con acero tipo A-36. Los tendones de postensado se consideraron de acero con una resistencia última especificada, $f_{p u}$, igual a $1670 \mathrm{MPa}$.

El espectro de diseño adoptado fue el de la NTCS del Estado de Guerrero (RCEG, Reglamento de Construcciones para los Municipios del Estado de Guerrero, 1989), suelo firme (tipo I), para la zona de Acapulco (zona D) y edificaciones tipo B (figura 3). La ordenada espectral fue la máxima correspondiente a la zona plana del espectro. El factor de comportamiento sísmico empleado $(Q)$ fue igual a 2 y la distorsión relativa de entrepiso máxima especificada fue 0.012. El coeficiente sísmico de diseño fue igual a 0.25 .

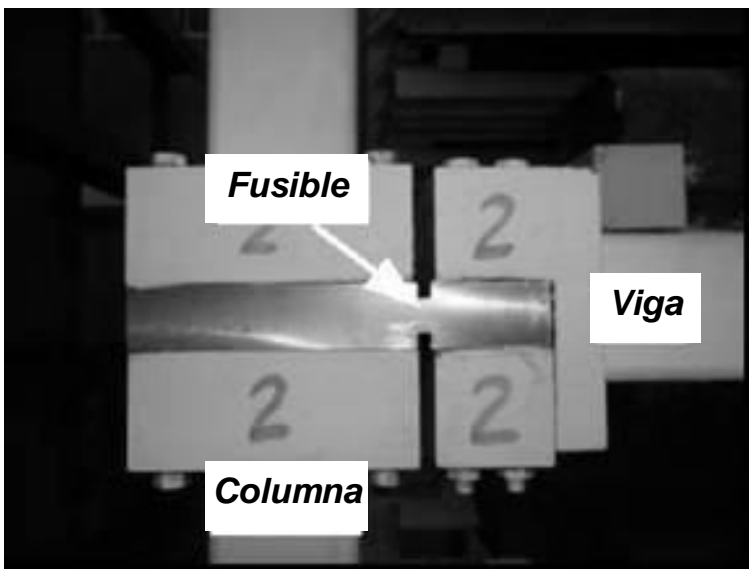

Figura 2. Detalle de fusible en unión viga-columna

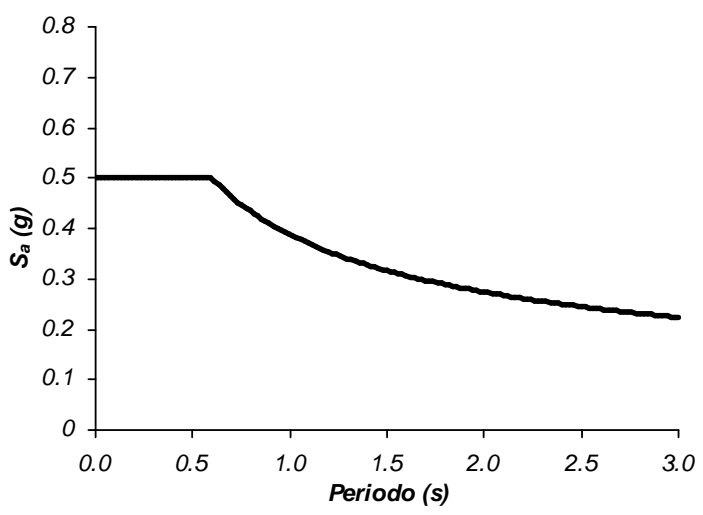

Figura 3. Espectro de diseño. Zona de Acapulco, suelo tipo I, edificaciones tipo B

En el espécimen E1, las dimensiones del muro convencional se obtuvieron considerando el criterio de diseño de que el muro tomara $60 \%$ del cortante total en la base, esto produjo que la participación calculada del muro fuese igual al 29\% del momento de volteo total de diseño. Para los especímenes A1 y A2 la solicitación sísmica para el diseño de los muros se definió asignando al momento de volteo de éstos 
un porcentaje del momento de volteo del sistema. Esto llevó a una participación de los muros de 30\% del momento total en el espécimen A1 y una participación de 60\% en el espécimen A2. Para lograr estos porcentajes fue necesario emplear en el edificio dos muros en el espécimen A2, mientras que en los otros especímenes (E1 y A1) se empleó sólo un muro.

Los ensayes realizados en los tres especímenes fueron: vibración ambiental, vibración libre, sismo de baja intensidad (comportamiento elástico), sismo de alta intensidad (intensidad de diseño) y sismo de muy alta intensidad (intensidad máxima aplicada).

Una descripción detallada de la instrumentación empleada en los diferentes ensayes en mesa vibradora de los especímenes estudiados se encuentra en Salinas (2013). Los desplazamientos laterales relativos a la base en cada nivel de los especímenes se midieron con transductores de desplazamiento digitales. Las rotaciones de los fusibles en las vigas se midieron también con transductores de desplazamiento digitales. Las aceleraciones absolutas en la dirección de la aplicación del movimiento sísmico se midieron con dos acelerómetros ubicados en cada nivel de los especímenes, además en el último nivel se colocó un acelerómetro en la dirección transversal del movimiento con el fin de detectar posibles efectos de torsión, los que se encontraron fueron despreciables en todos los casos.

Adicionalmente, se instalaron celdas de carga para la medición de las variaciones de la fuerza en los cables de postensado, además de deformímetros en las bielas que formaban parte de la armadura de conexión entre el marco y los muros. También se instrumentó, con deformímetros eléctricos, las secciones críticas de las barras disipadoras dentro de la cimentación (Salinas, 2013).

\section{Geometría y materiales}

\section{Espécimen E1}

El sistema estructural del espécimen E1 fue del tipo dual, en el que se combinó dos marcos de acero y un muro convencional, este último diseñado para tener una participación del $60 \%$ del cortante total de diseño en la base, que implicó una participación del 29\% del momento de volteo total de diseño (Sánchez, 2008). Antes de los ensayes se obtuvieron características de las propiedades mecánicas de los materiales usados, cuyos resultados se muestran en la tabla 1. Como se aprecia en esta tabla, el acero de refuerzo del muro de este espécimen correspondió a varillas corrugadas de $6 \mathrm{~mm}$ de diámetro, las que se importaron de Nueva Zelandia, ya que refuerzo corrugado con este diámetro no se produce en México. El esfuerzo de fluencia de este refuerzo fue 480.2 MPa (tabla 1), valor cercano a los que tendrían aceros de refuerzo producidos en México. El peso total del espécimen fue de $67.9 \mathrm{kN}$. El muro tenía una sección de $0.25 \times 0.08 \mathrm{~m}$, y una altura de $3000 \mathrm{~mm}$, figura 4. En dicha figura, se presentan los esquemas de elevación y planta del espécimen, así como la sección transversal con el refuerzo del muro, con indicación de los pesos en cada piso. La unión entre el muro y los marcos se logró mediante el empleo de una armadura rígida en cada uno de los cinco niveles, figura 4. Esta armadura se conectaba al muro y los marcos mediante un sistema de articulaciones, de manera de representar el comportamiento que tendría una barra con sólo carga axial en ésta (Sánchez, 2008).

\section{Espécimen A1}

El sistema estructural del espécimen A1 fue del mismo tipo que el comentado para el espécimen E1, con la variante de que tenía un muro autocentrado diseñado para tener una participación del $30 \%$ del momento de volteo total de diseño La sección del muro era $0.35 \times 0.09 \mathrm{~m}$, con una altura de $3000 \mathrm{~mm}$ (figura 5). El sistema de unión en cada nivel, entre los marcos y muro, fue el mismo anteriormente 
descrito para el espécimen E1. Además, el muro tenía dos tendones de postensado de $6 \mathrm{~mm}$ de diámetro y dos barras disipadoras de $6 \mathrm{~mm}$ de diámetro, construidas empleando dos barras corrugadas convencionales, producidas en México, de $9.5 \mathrm{~mm}$ de diámetro. Estas barras se maquinaron en una longitud de $65 \mathrm{~mm}$ para generar el disipador tipo dog-bone de superficie lisa, figura 5. La fuerza de postensado aplicada en cada tendón estuvo asociada al valor $0.45 f_{p u}$. El peso total del espécimen fue igual a $67.9 \mathrm{kN}$. El tipo de acero de refuerzo longitudinal para el muro de este espécimen fue el mismo comentado para el espécimen E1, con las propiedades mecánicas mostradas en la tabla 1. En la figura 5 se presentan los esquemas de elevación y planta del espécimen, así como la sección transversal del muro, con indicación de los pesos en cada piso.

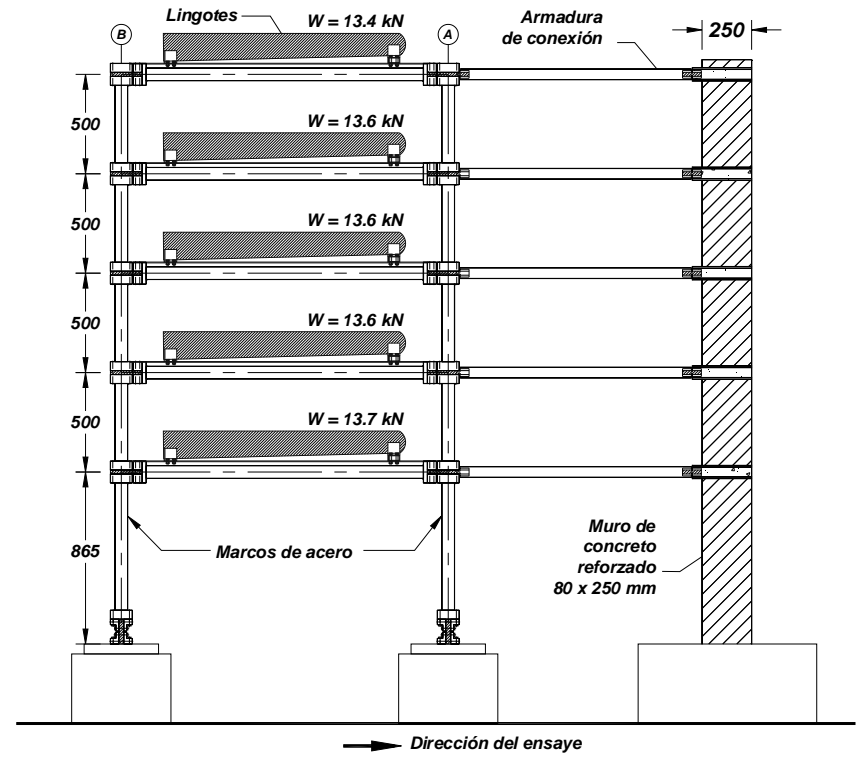

(a) Elevación

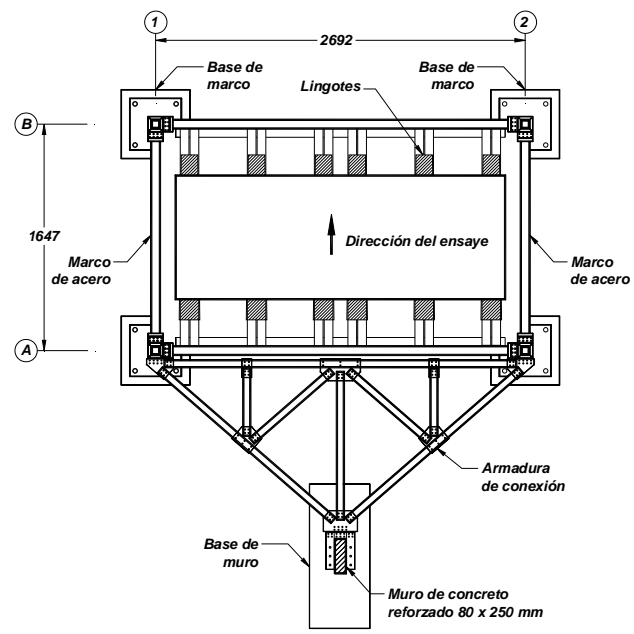

(b) Planta

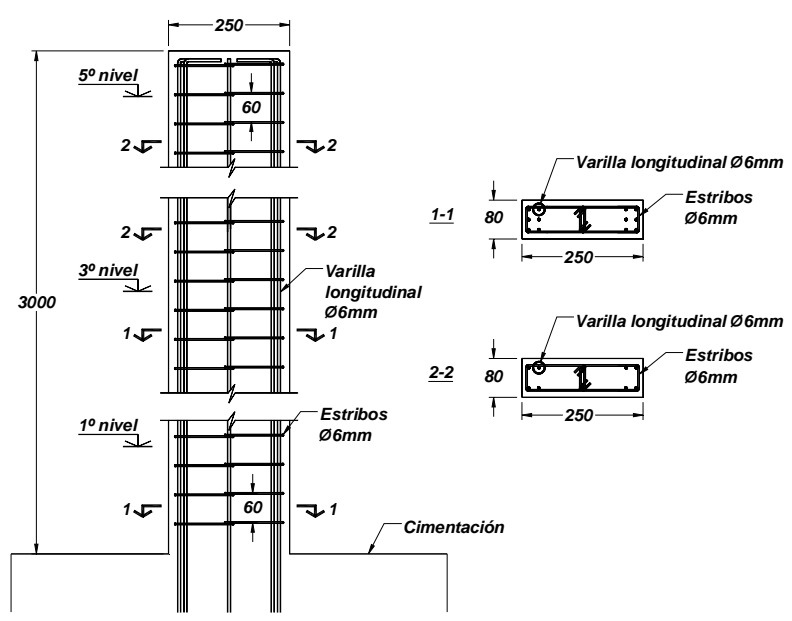

(c) Detalles de refuerzo y sección transversal

Figura 4. Esquemas del espécimen E1, dimensiones en mm (adaptado de Sánchez, 2008) 


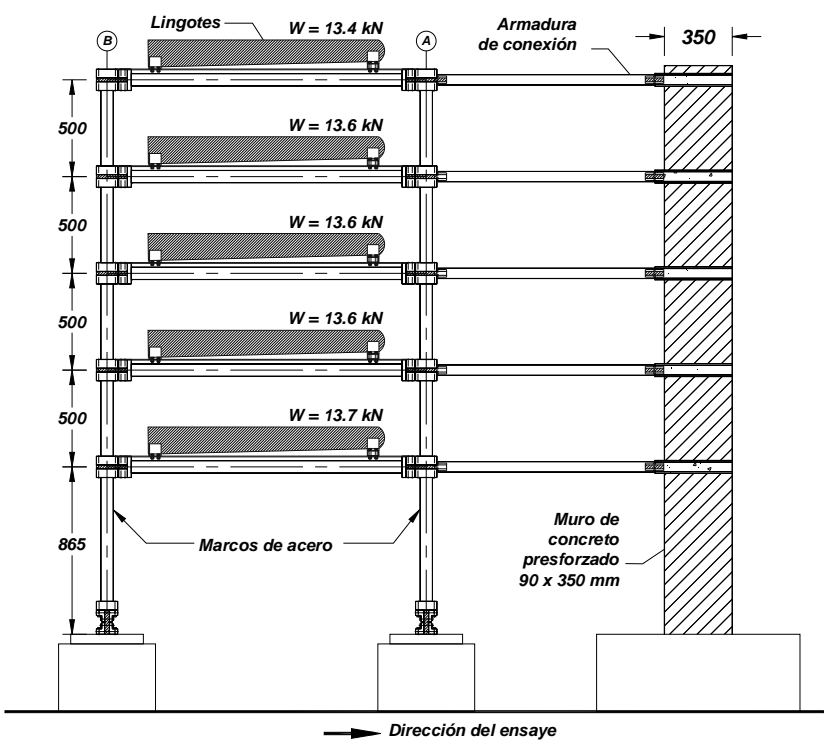

(a) Elevación

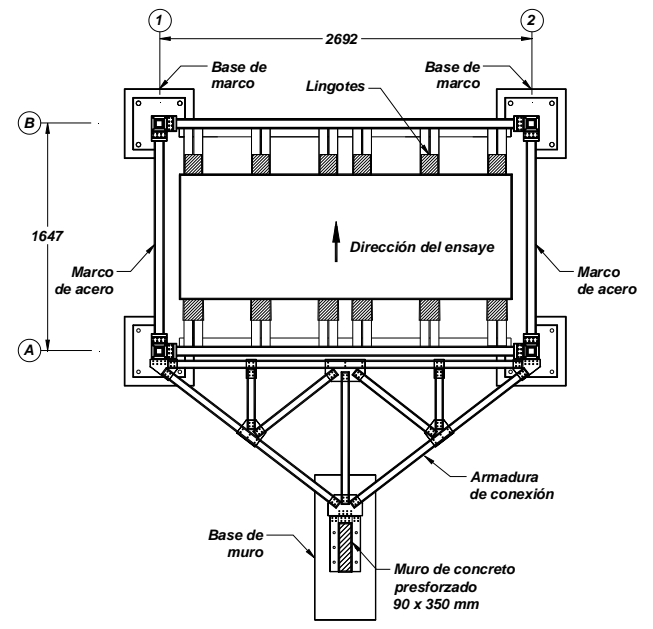

(b) Planta
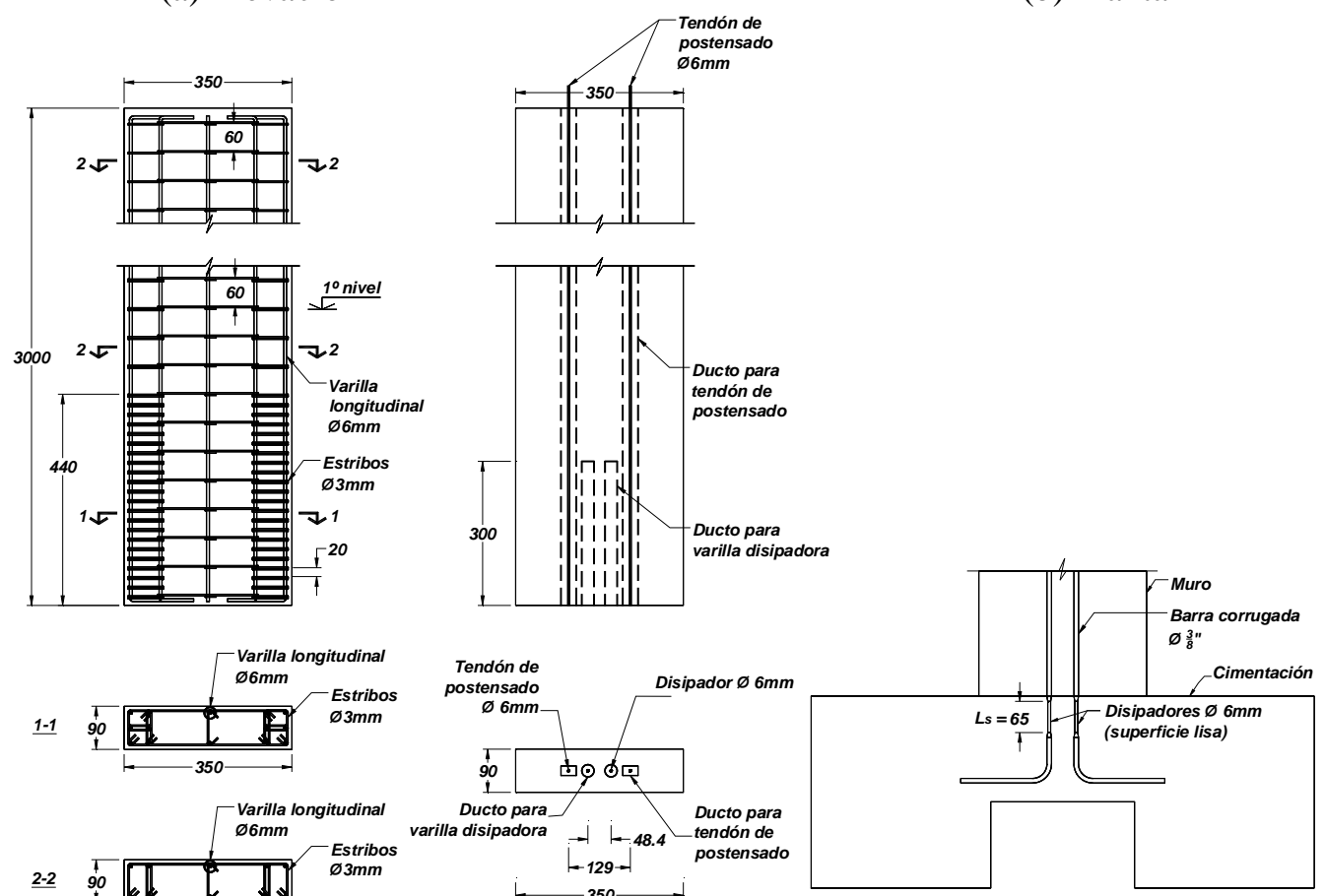

$\underline{2-2}$
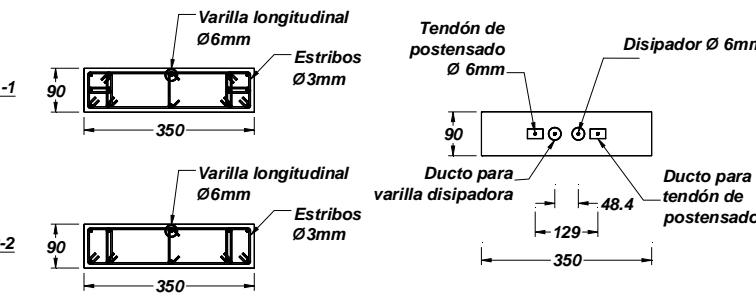

(c) Detalles de refuerzo y secciones transversales del muro, y de las barras disipadoras

Figura 5. Esquemas del espécimen A1, dimensiones en mm

\section{Espécimen A2}

El sistema estructural del espécimen A2 era del mismo tipo del anteriormente descrito para el espécimen A1, con la variante de emplear dos muros autocentrados, en lugar de solo uno. Estos muros se diseñaron de manera tal que en conjunto tomaran el $60 \%$ en el momento de volteo total del sistema. Esto se hizo con el fin de estudiar el efecto de diferentes contribuciones de muros en un sistema dual cuando responde a acciones sísmicas. La sección de cada muro era $0.55 \times 0.09 \mathrm{~m}$, con la misma altura de los muros 
E1 y A1. Los dos tendones de postensado eran de $7 \mathrm{~mm}$ de diámetro. La fuerza de postensado aplicada en cada tendón estuvo asociada al valor $0.30 f_{p u}$. Además, se emplearon dos barras disipadoras de $7 \mathrm{~mm}$ de diámetro. Estas barras fueron preparadas empleando barras corrugadas convencionales producidas en México de $12.7 \mathrm{~mm}$ de diámetro, maquinadas en una longitud de $100 \mathrm{~mm}$ para generar el disipador tipo dog-bone de superficie lisa. El tipo de acero de refuerzo longitudinal para los muros de este espécimen fue el mismo comentado para el espécimen E1, con las propiedades mecánicas mostradas en la tabla 1. El peso total del espécimen era $73.7 \mathrm{kN}$. En la figura 6 se presentan la elevación y la planta del espécimen A2, así como la sección transversal del muro típico, con indicación de los pesos en cada piso.

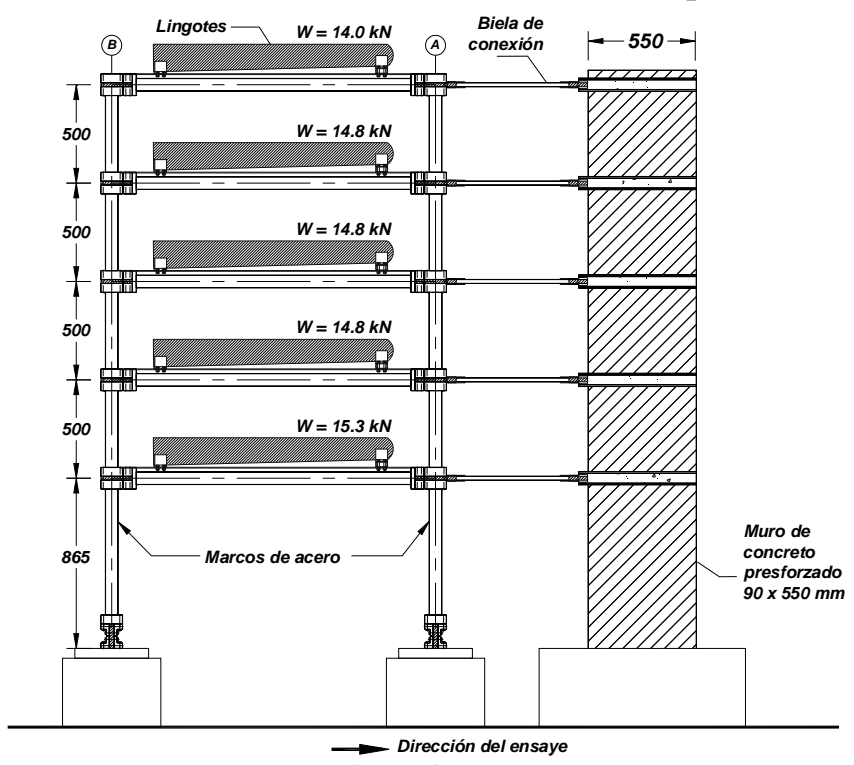

(a) Elevación

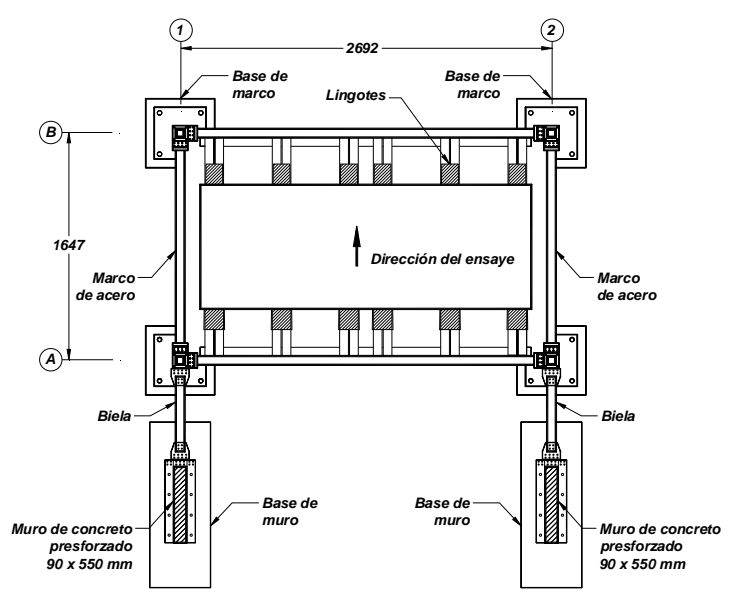

(b) Planta

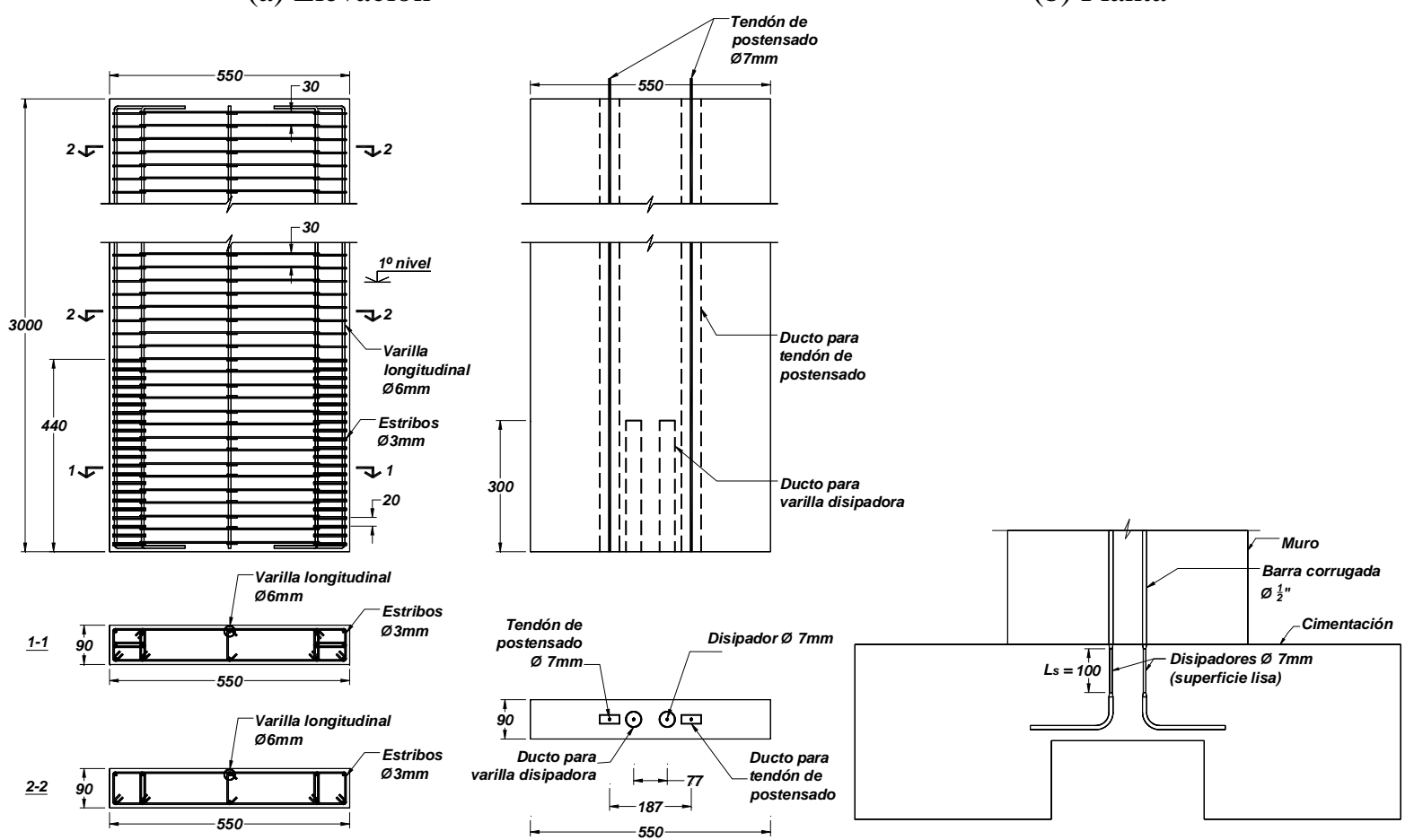

(c) Detalles de refuerzo y secciones transversales del muro, y de las barras disipadoras

Figura 6. Esquemas del espécimen A2, dimensiones en mm 
Tabla 1. Propiedades mecánicas de los materiales usados en los especímenes, medidas en el tiempo de ensaye

\begin{tabular}{cccc}
\hline Propiedad del material & Esp. E1 & Esp. A1 & Esp. A2 \\
\hline Resistencia a la compresión del concreto (MPa) & 47.1 & 44.1 & 48.1 \\
Esfuerzo de fluencia del acero de refuerzo del muro (MPa) & 480.2 & 480.2 & 480.2 \\
Esfuerzo de fluencia de disipadores (MPa) & - & 431.6 & 431.6 \\
Esfuerzo de fluencia de los fusibles del marco (MPa) & 307.7 & 313.9 & 269.8 \\
Esfuerzo de rotura del acero de postensado (MPa) & -- & 1802.1 & 1758.0 \\
\hline
\end{tabular}

\section{Ensayes realizados}

En los ensayes de vibración ambiental y de vibración libre (Salinas, 2013), un resultado relevante es la frecuencia fundamental del espécimen, la cual se obtiene mediante la función de transferencia entre el registro de aceleraciones del piso 5 y el del movimiento en la base.

Para los ensayes en mesa vibradora se empleó el acelerograma registrado en la estación Llolleo, componente EO, el 3 de marzo de 1985 en Chile, por ser un acelerograma de un sismo intenso, dominante en períodos cortos y que inducía un comportamiento inelástico importante en los especímenes de ensaye. Además, el espectro elástico del registro sin escalar es comparable en algunas zonas del espectro con el espectro de diseño de la zona de Acapulco (zona D) y tipo de terreno I, especificado por el Reglamento de Construcciones para los Municipios del Estado de Guerrero (RCEG, 1989), multiplicado por un factor de sobrerresistencia igual a 2, valor que toma en cuenta la sobrerresistencia implícita en una estructura diseñada de acuerdo con normativas en México. La sobrerresistencia se define como el cociente entre la resistencia de la estructura y la calculada con procedimientos convencionales elásticos empleando los requisitos de diseño correspondientes de la normativa que se emplee. El registro no fue escalado porque los especímenes no fueron diseñados a escala y no se emplearon leyes de similitud. La figura 7a muestra el registro sísmico de Llolleo, cuya aceleración máxima es $0.64 \mathrm{~g}$. La figura $7 \mathrm{~b}$ muestra el espectro elástico de aceleración del registro usado, para una fracción de amortiguamiento crítico igual a 5\%, así como el espectro elástico de diseño del RCEG (1989), multiplicado por el valor de la sobrerresistencia. Para el intervalo de periodos de interés, se consideró apropiado el uso del registro de Llolleo ya que para los periodos fundamentales de los especímenes, que son del orden de $0.25 \mathrm{~s}$ y $0.40 \mathrm{~s}$, implicarían considerar una sobrerresistencia del orden de 2.7 a 3, lo cual está dentro del intervalo de 2 a 3 que se menciona en la literatura (Uang, 1991; Rodríguez y Restrepo, 2012).

\section{Sismo de alta intensidad (intensidad de diseño)}

El registro original para el espécimen E1 fue filtrado con un filtro pasa-banda de 0.2 y $30 \mathrm{~Hz}$ que, sumado a factores de ruido en el funcionamiento de la mesa vibradora, produjo aceleraciones máximas mayores que los del registro objetivo, del orden de 0.93g (Sánchez, 2008); éste fue el único movimiento de alta intensidad aplicado al espécimen. Para el espécimen A1, el registro original fue filtrado con un filtro pasa-banda de 0.2 y $15 \mathrm{~Hz}$, que produjo aceleraciones máximas reales menores en el registro medido, del orden de $0.61 \mathrm{~g}$. Para el espécimen A2, el registro original fue filtrado con un filtro pasa-banda de $0.5 \mathrm{y}$ $30 \mathrm{~Hz}$ que, sumado a factores relativos a la operación de la mesa vibradora, produjo una mayor duración del movimiento en esta fase y aceleraciones ligeramente mayores a las del registro medido, del orden de $0.71 \mathrm{~g}$. La figura 8 muestra los espectros elásticos de pseudoaceleraciones, $S_{a}$, de los registros medidos y objetivos, calculados considerando una fracción de amortiguamiento crítico igual a $5 \%$. 


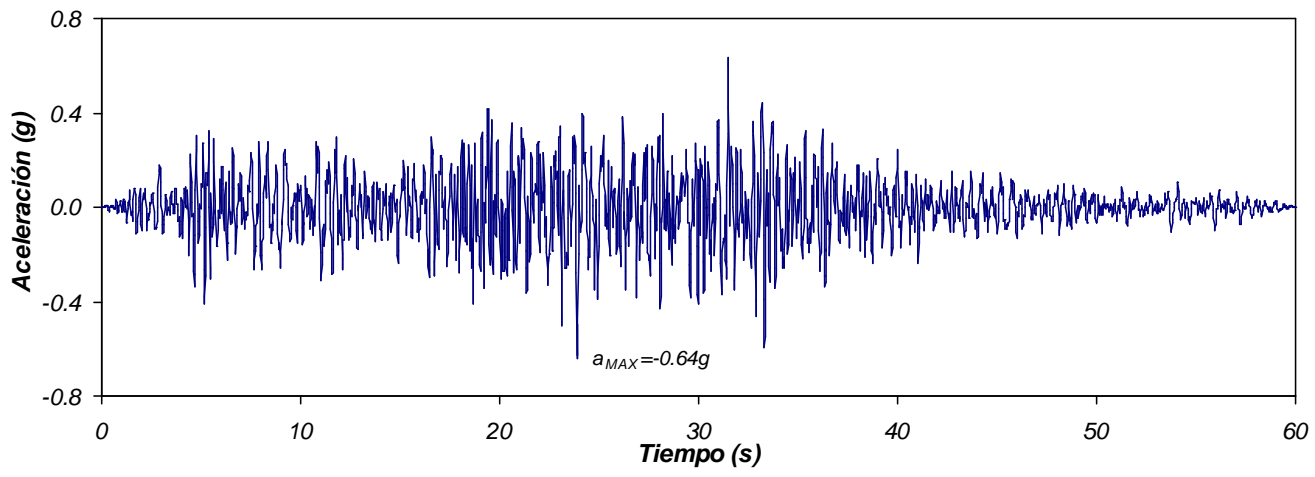

(a) Acelerograma de Llolleo, 1985

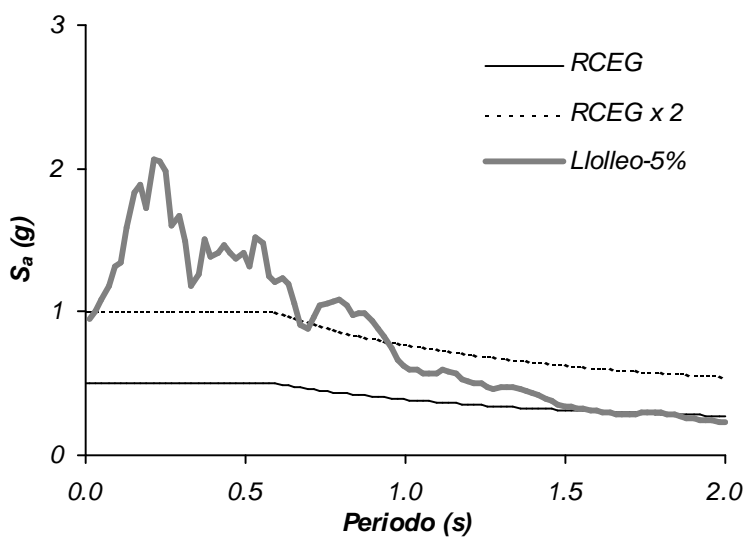

(b) Espectros de aceleración y de diseño

Figura 7. Registro y espectros de aceleración de Llolleo y espectro de diseño del RCEG (1989)

\section{Sismo de muy alta intensidad (intensidad máxima aplicada)}

Para el espécimen A1, el movimiento en la base empleado para el espécimen A1 fue el registro de aceleraciones de Llolleo escalado por un factor de 1.50, con una aceleración máxima objetivo de $0.98 g$. Como en el caso anterior, el registro original fue filtrado con un filtro pasa-banda de 0.2 y $15 \mathrm{~Hz}$, que produjo en el registro medido una aceleración máxima del orden de $1.00 g$. Para el espécimen A2, el movimiento en la base empleado fue el registro de aceleraciones de Llolleo escalado por un factor de 1.25, con una aceleración máxima de $0.81 \mathrm{~g}$; el registro original fue filtrado con un filtro pasa-banda de 0.5 y 30 $\mathrm{Hz}$ que, sumado a factores de ruido en el funcionamiento de la mesa vibradora, produjo en el registro medido en la mesa aceleraciones ligeramente mayores que las del registro objetivo, con una aceleración máxima de $0.89 \mathrm{~g}$. La figura 9 permite comparar los espectros elásticos de pseudoaceleraciones de los registros medidos y objetivos en esta etapa de ensaye, calculados considerando una fracción de amortiguamiento crítico igual a 5\%, valor comúnmente empleado para definir espectros de diseño en diferentes normativas de construcción. 


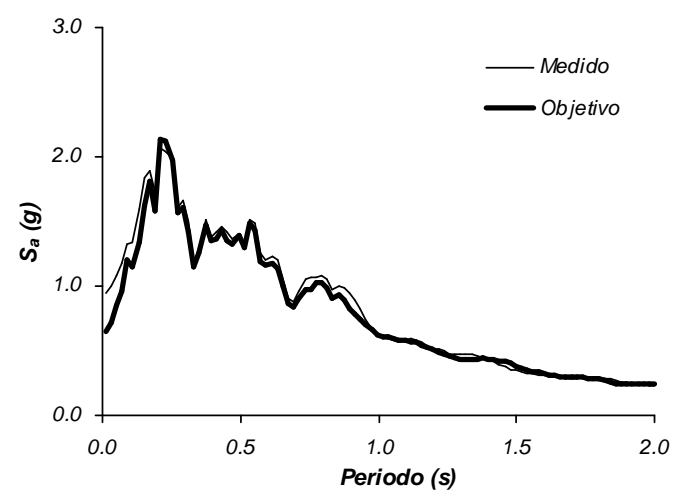

(a) Espécimen E1

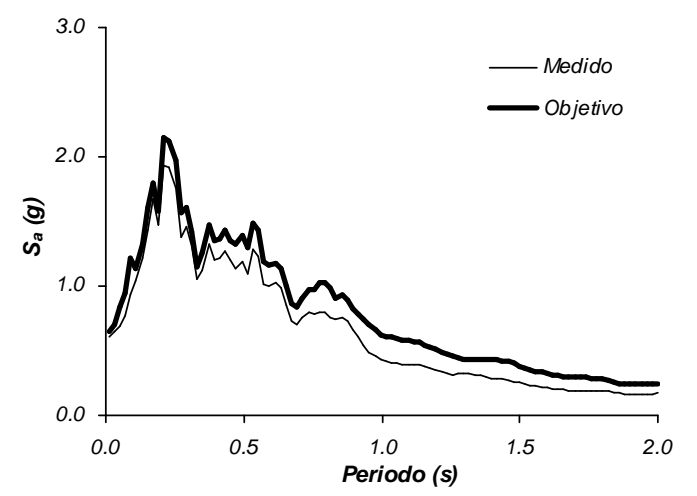

(b) Espécimen A1

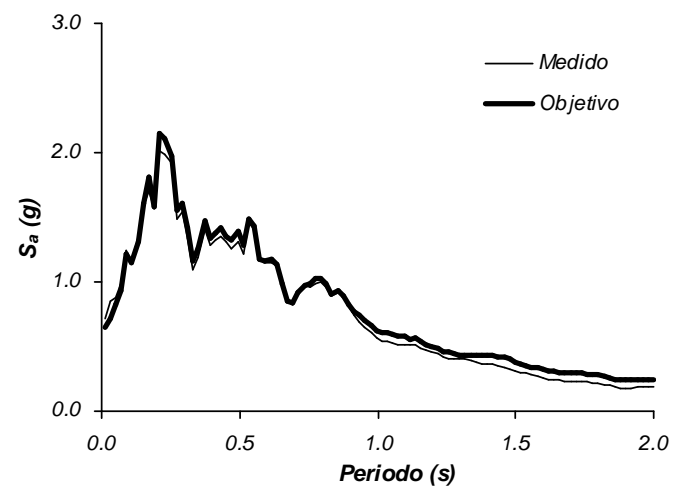

(c) Espécimen A2

Figura 8. Espectros elásticos de pseudoaceleración $(\xi=5 \%)$ de los registros medidos y objetivos del movimiento de la base. Sismos de intensidad de diseño

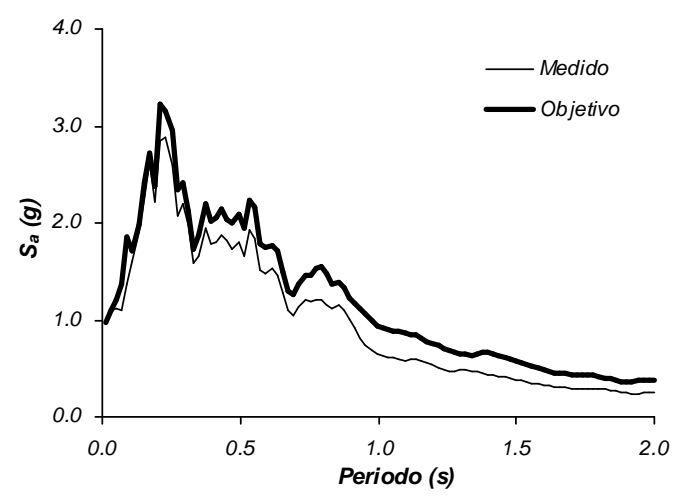

(a) Espécimen A1

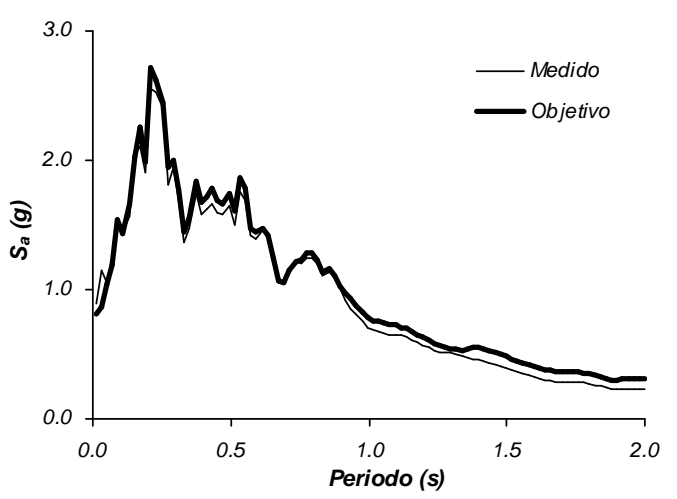

(b) Espécimen A2

Figura 9. Espectros elásticos de pseudoaceleración $(\xi=5 \%)$ de los registros medidos y objetivos del movimiento de la base. Sismos de intensidad máxima aplicada

Para comparar la intensidad sísmica de cada registro medido, se calculó la intensidad de Arias de cada registro, $I_{A S M}$, y se dividió entre la intensidad de Arias del registro objetivo original sin escalar, $I_{A S O}$, por lo que se obtuvieron intensidades sísmicas normalizadas para los sismos de intensidad de diseño y de intensidad máxima aplicada, empleados en los especímenes, que se presentan en la figura 10. Con este procedimiento, los sismos de intensidad de diseño (ID) para los especímenes A1, A2 y E1 tuvieron intensidades sísmicas de $0.79,1.39$ y 1.11 veces la intensidad del sismo objetivo, respectivamente. Los 
sismos de intensidad máxima aplicada (IMA) para los especímenes A1 y A2 tuvieron intensidades sísmicas de 1.80 y 1.45 veces la intensidad del sismo objetivo, respectivamente. Se debe mencionar que el espécimen E1 no se ensayó con el sismo de intensidad máxima por limitaciones de tiempo en el programa de investigación.

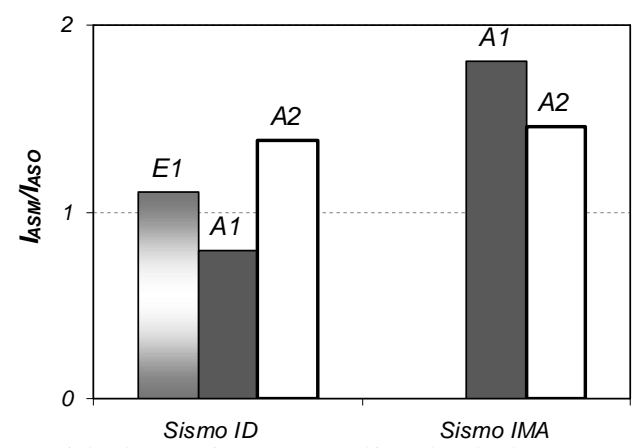

Figura 10. Intensidad sísmica normalizada de los ensayes realizados

\section{EVALUACIÓN DE RESULTADOS}

La figura 11 muestra las envolventes de aceleraciones medidas de piso divididas entre la aceleración máxima de la base, versus la altura relativa de piso (definida como el cociente entre la altura de piso y la altura total del edificio), para los sismos empleados. Los especímenes A1 y E1 tienen un perfil de envolventes parecido (figura 11a), salvo por la amplificación en el último piso, mayor en el caso del espécimen E1, debido a una falla local en la armadura que formaba la conexión entre el marco y el muro en el último piso. La figura 12 muestra las envolventes de desplazamientos laterales relativos a la base, $\Delta_{i}$, divididos entre la altura total del edificio, $H$. Estos desplazamientos están en función de la altura relativa de piso. La relación $\Delta_{i} / H$ para el nivel de azotea se conoce también como distorsión global. Para los sismos de intensidad de diseño, las distorsiones globales máximas fueron iguales a $3.6 \%, 2.8 \%$ y $2.9 \%$ en los especímenes E1, A1 y A2, respectivamente. Para los sismos de intensidad máxima aplicada, las distorsiones globales máximas fueron iguales a $4.1 \%$ en los especímenes A1 y A2. En particular, la forma lineal de las envolventes de desplazamientos de los especímenes A1 y A2 sugiere que la distorsión de entrepiso máxima puede aproximarse a la distorsión global del edificio.

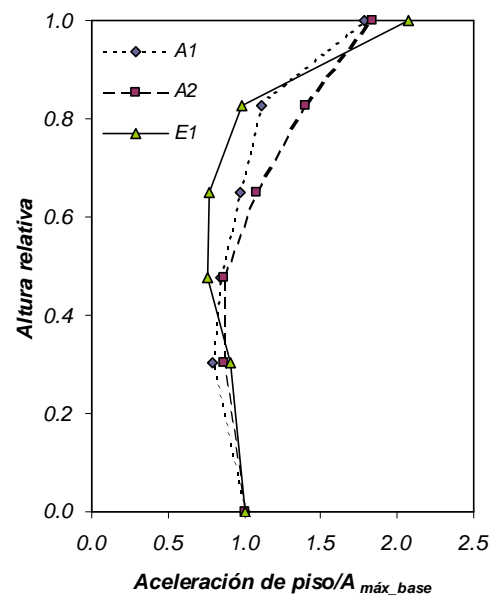

(a) Intensidad de diseño

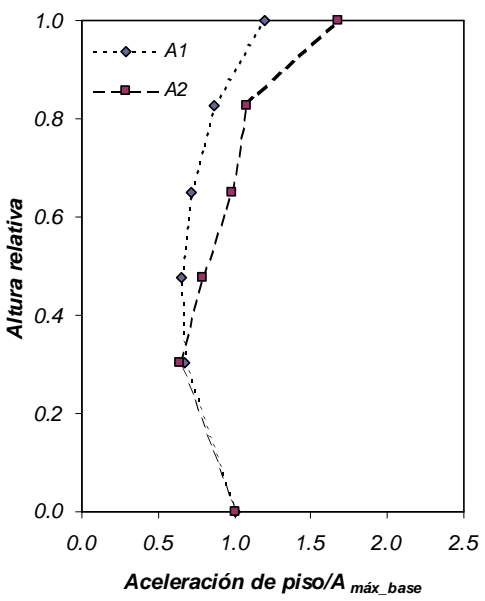

(b) Intensidad máxima aplicada

Figura 11. Envolvente de aceleraciones de piso 


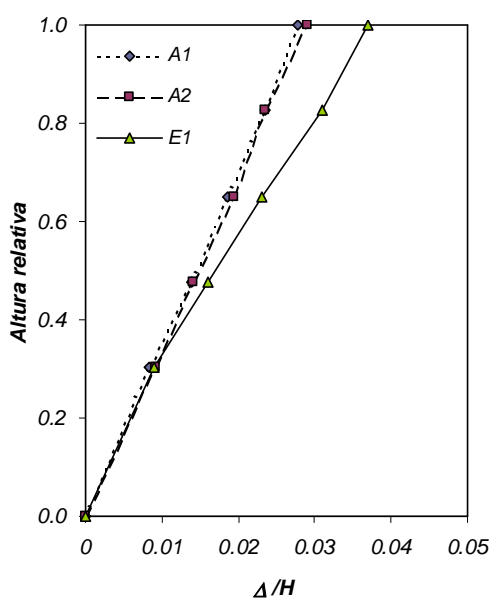

(a) Intensidad de diseño

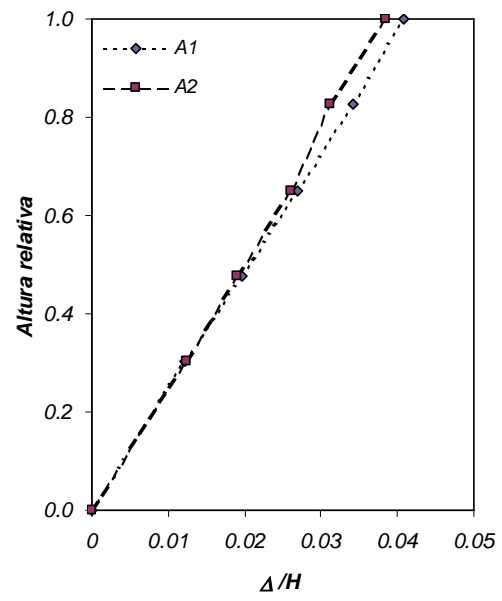

(b) Intensidad máxima aplicada

Figura 12. Envolvente de desplazamientos laterales relativos a la base, $\Delta_{i}$, divididos entre la altura total del edificio, $H$

El desempeño sísmico de los especímenes en el comportamiento inelástico fue evaluado mediante el cálculo del factor $R_{M}$, que es un parámetro que toma en cuenta la ductilidad global y el efecto de la sobrerresistencia de la estructura, $\Omega_{o}$, donde este parámetro se define como el cociente entre el cortante basal resistente y el de diseño. El factor $R_{M}$ representa un factor de reducción de la respuesta sísmica poco afectado por los efectos de los modos superiores (Rodríguez et al., 2007) y está definido por la expresión:

$R_{M}=\frac{M_{v e}}{M_{v o}}$

donde $M_{v e}$ y $M_{v o}$ son los momentos de volteo máximos por sismo en el edificio calculado manteniendo una respuesta elástica y aquél que se obtiene de la respuesta inelástica no lineal calculada, respectivamente.

La sobrerresistencia, $\Omega_{o}$, fue calculada a partir de una estimación del cortante máximo asociado al modo fundamental de vibración, $V_{o}$, definido por la expresión:

$V_{o}=\Omega_{o} C_{S} W$

donde $C_{S}$ es el coeficiente sísmico empleado para el diseño del edificio. $V_{o}$ se puede calcular de manera aproximada con el cociente:

$V_{o}=\frac{M_{v o}}{H_{e f}}$

donde $M_{v o}$ es el momento de volteo máximo en la base calculado con las aceleraciones horizontales medidas en el edificio, y $H_{e f}$ es la altura efectiva del primer modo de vibración de la estructura, obtenida de la forma modal experimental de dicho modo. La ec. 3 está basada en la hipótesis de que el momento de volteo no está afectado por los modos superiores y que la altura efectiva del primer modo no cambia 
significativamente durante la respuesta inelástica. A partir de las ecs. 2 y 3, se obtiene una expresión para la sobrerresistencia:

$$
\Omega_{o}=\frac{V_{o}}{C_{S} W}
$$

Con las mediciones experimentales de los ensayes de intensidad de diseño, para los especímenes E1, A1 y A2 se calcularon valores del parámetro $R_{M}$ iguales a 2.2, 3.0 y 2.5, respectivamente. Estos valores evidencian que los especímenes incursionaron en el intervalo de comportamiento inelástico. Además, estos valores son mayores que el valor del factor de comportamiento sísmico empleado para el diseño de estos especímenes, valor comentado anteriormente. Para los sismos de intensidad máxima aplicada, los valores experimentales del parámetro $R_{M}$ fueron iguales a 4.4 y 3.0 para los especímenes A1 y A2, respectivamente, lo cual es un indicador de una mayor incursión en el intervalo de comportamiento inelástico que alcanzaron los especímenes.

Para el sismo de intensidad de diseño los valores de $R_{M}$ obtenidos con las mediciones experimentales en los especímenes E1 y A1 fueron diferentes, debido a la mayor magnitud de la aceleración registrada en el último piso del espécimen E1 asociada a una falla local de la biela entre el marco y el muro. Mediante un análisis dinámico no lineal del modelo del espécimen E1, cuyos detalles se presentan en la sección siguiente, se calculó un valor de $R_{M}$ igual a 3.2. En el caso del espécimen E1, fueron adoptados los parámetros $R_{M}$ y $\Omega_{o}$ calculados con el modelo analítico, basado en la comparación de las historias de desplazamientos y aceleraciones medidas con las calculadas y la detección de la falla local mencionada en último piso del espécimen.

La figura 13 presenta espectros de piso, es decir, espectros de respuesta de pseudoaceleración $\left(S_{a}\right)$ para el último piso de los especímenes ensayados, divididos entre la aceleración máxima de la base (PGA). Los espectros fueron calculados a partir de la respuesta de osciladores elásticos con una fracción de amortiguamiento igual a $2 \%$ sometidos a un movimiento de la base definido por la aceleración medida en el último piso de cada espécimen. Este valor es menor que el comúnmente empleado para espectros de diseño, y su empleo se basa en resultados de ensayes de un edificio de siete niveles en mesa vibradora, los cuales mostraron que el emplear en análisis dinámicos no lineales valores de la fracción de amortiguamiento crítico mayores que $2 \%$ lleva a subestimar de manera importante los desplazamientos laterales de la estructura (Panagiotou, 2008; Martinelli y Filippou, 2009). En cada figura se muestran dos espectros; la línea delgada continua representa la respuesta elástica del espécimen y la línea gruesa representa el espectro de respuesta correspondiente a la respuesta inelástica del espécimen; además se indican, en línea vertical discontinua, los periodos de los dos primeros modos de vibración de las estructuras obtenidas con la respuesta elástica de los especímenes. La magnitud de la reducción de la respuesta se valuó con el cociente entre los valores espectrales de pseudoaceleración obtenidos para la respuesta elástica y aquellos obtenidos para la respuesta inelástica, en los periodos predominantes de las estructuras considerando la elongación de los periodos que ocurre como consecuencia de la acción sísmica. Los periodos predominantes en el comportamiento inelástico fueron iguales a $0.49 \mathrm{~s}, 0.49 \mathrm{~s}$ y $0.45 \mathrm{~s}$, en los especímenes E1, A1 y A2, respectivamente, mientras que en el comportamiento elástico fueron iguales a $0.41 \mathrm{~s}, 0.38 \mathrm{~s}$ y $0.26 \mathrm{~s}$ en los mismos especímenes. En la figura 14 se muestran para el periodo fundamental los valores calculados del cociente entre los valores espectrales elásticos e inelásticos mostrados en la figura 13, que confirman las observaciones realizadas a partir de los espectros de respuesta. No fue posible establecer con certeza la magnitud de la reducción en el segundo modo, debido a la dificultad de definir la contribución de este modo de vibración en los espectros de piso obtenidos con la respuesta inelástica. Además, se debe observar que la frecuencia del segundo modo de vibración del espécimen A2 fue mayor 
que la frecuencia máxima del registro filtrado, igual a $30 \mathrm{~Hz}$, por lo que el comportamiento capturado en las mediciones obtenidas en los ensayes de este espécimen correspondió sólo al primer modo de vibración.

Los valores de la sobrerresistencia fueron valuados para cada etapa de comportamiento inelástico, empleando los resultados experimentales para los especímenes A1 y A2, y empleando los resultados analíticos para el espécimen E1. Para los sismos de intensidad de diseño, la sobrerresistencia $\Omega_{o}$ en los especímenes E1, A1 y A2 fue igual a 2.0, 2.1 y 2.9, respectivamente. Para los sismos de intensidad máxima aplicada, la sobrerresistencia $\Omega_{o}$ en los especímenes A1 y A2 fue igual a 2.3 y 3.0, respectivamente.

Se aprecia que los especímenes A1 y E1 alcanzaron sobrerresistencias y factores $R_{M}$ de similar magnitud, teniendo en ambos casos una participación similar de los muros en la respuesta estructural del sistema total. Esto sugiere la posibilidad de establecer, para los sistemas muro-marco con muros autocentrados, criterios de diseño sísmico similares a los empleados en los sistemas con muros convencionales, lo que es analizado en otro trabajo (Salinas y Rodríguez, 2013). El espécimen A2 alcanzó una sobrerresistencia mayor, asociada a una mayor participación de los muros en la respuesta estructural total.

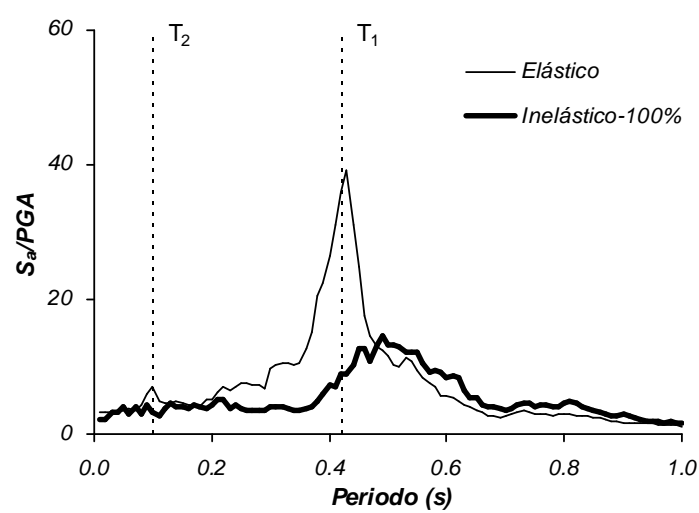

(a) Espécimen E1

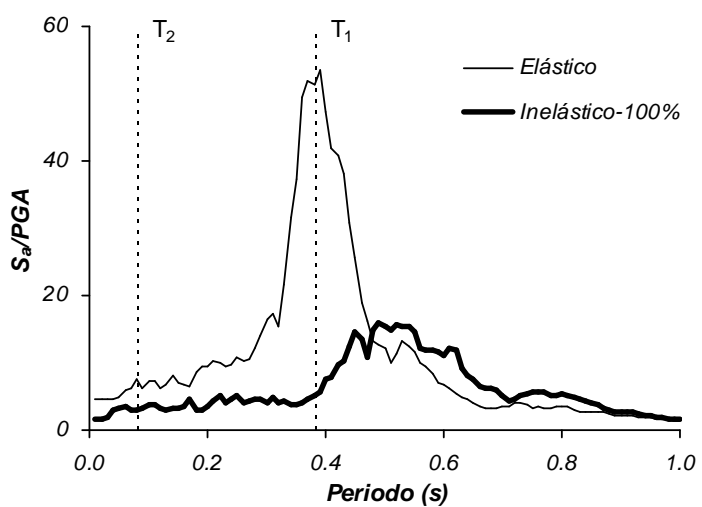

(b) Espécimen A1

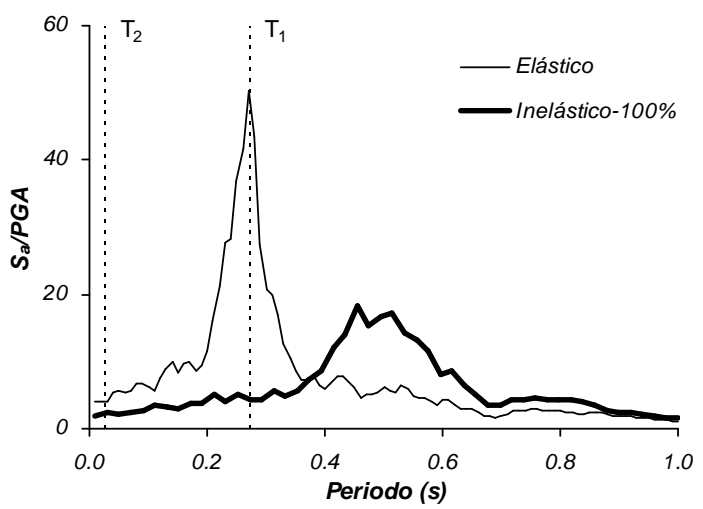

(c) Espécimen A2

Figura 13. Espectros normalizados de aceleración del movimiento en el piso 5. Movimientos de baja intensidad (comportamiento elástico) y alta intensidad (comportamiento inelástico) 


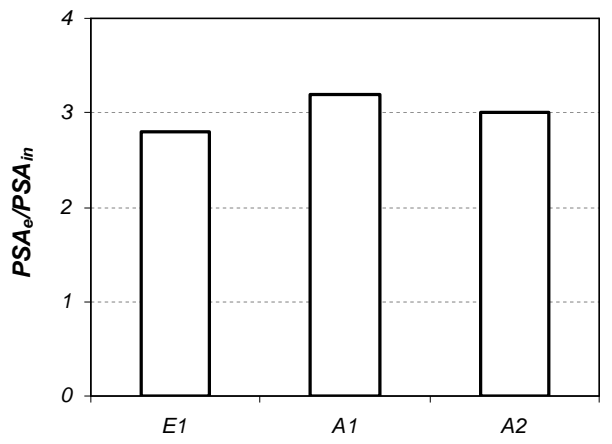

Figura 14. Cociente de valores espectrales de pseudoaceleración para los especímenes

La figura 15a muestra las distorsiones máximas de entrepiso medidas en los especímenes versus la intensidad sísmica normalizada acumulada para cada espécimen. En este caso, la intensidad sísmica acumulada (la suma de las intensidades de cada movimiento) se considera un parámetro más completo que la intensidad sísmica particular para cada evento aislado, debido a que las deformaciones máximas y residuales en una estructura serán un resultado de la acumulación de la energía de entrada de cada evento sísmico en la estructura. La intensidad acumulada normalizada, $I_{A A N}$, se obtuvo dividiendo la intensidad de Arias acumulada entre la intensidad de Arias del sismo objetivo, $I_{A S O}$, por lo que sus valores para los especímenes A1 y A2 son mayores que los mostrados en la fig. 10. Se observa la menor deformabilidad del espécimen A2 en comparación a los especímenes A1 y E1, los cuales tienen una menor participación de los muros. La figura 15b muestra la relación entre la distorsión de entrepiso residual y la distorsión de entrepiso máxima para los especímenes, considerando todos los sismos a que fueron ensayados. Las distorsiones de entrepiso residuales medidas después del sismo de intensidad de diseño en los especímenes A1 y A2 fueron del orden de $0.19 \%$ y $0.17 \%$, respectivamente, mientras que para el espécimen E1 fue igual a $0.88 \%$. Como es de esperar, a mayor distorsión de entrepiso máxima, la distorsión residual aumenta; sin embargo, la distorsión residual en el espécimen con mayor participación de muros autocentrados es menor que la del espécimen con menor participación a medida que la intensidad sísmica aumenta. Después del sismo de intensidad máxima aplicada, la distorsión residual en el espécimen A2 fue igual a $0.30 \%$, mientras que en el espécimen A1 fue igual a $0.54 \%$, es decir 1.8 veces más. Esta característica observada a nivel del desempeño global de la estructura se refleja en las rotaciones permanentes de las secciones críticas de los marcos de los especímenes. Las rotaciones en los extremos de las vigas y en la base de las columnas se obtuvieron mediante las lecturas de potenciómetros adyacentes a estas secciones, como se muestra en la fig. 16a para una sección de viga. La figura 15c muestra las rotaciones residuales y las rotaciones máximas en la base de columna y en los extremos de las vigas de los marcos del segundo piso, para todos los eventos a que fueron ensayados los especímenes. Estos diagramas muestran que a medida que aumenta la participación de los muros autocentrados en la estructura, aumenta la capacidad de reducción de las deformaciones residuales. 


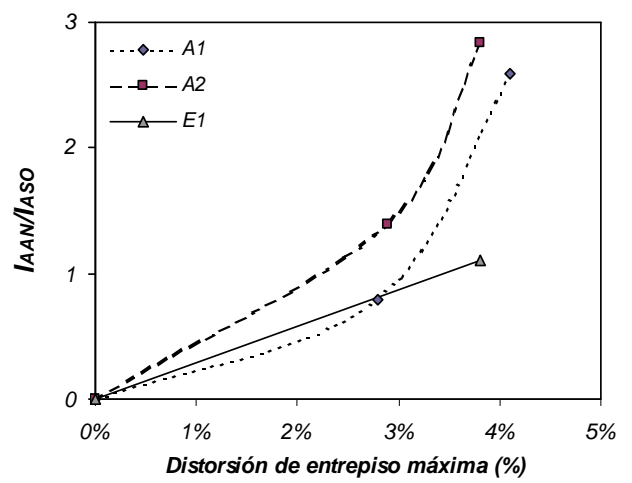

(a) Distorsión de entrepiso máxima.

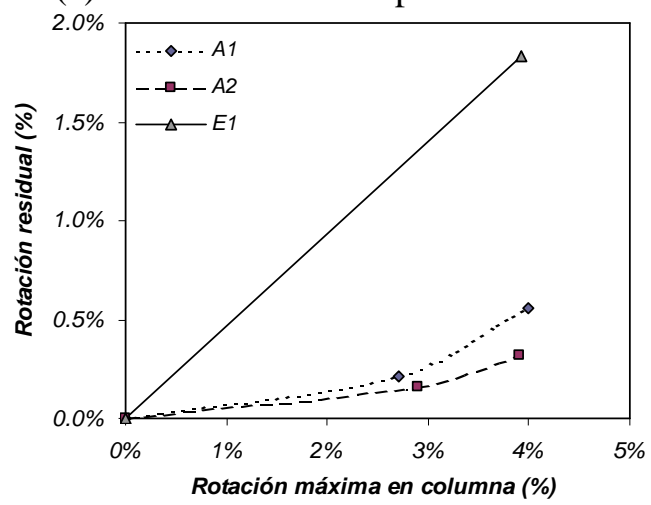

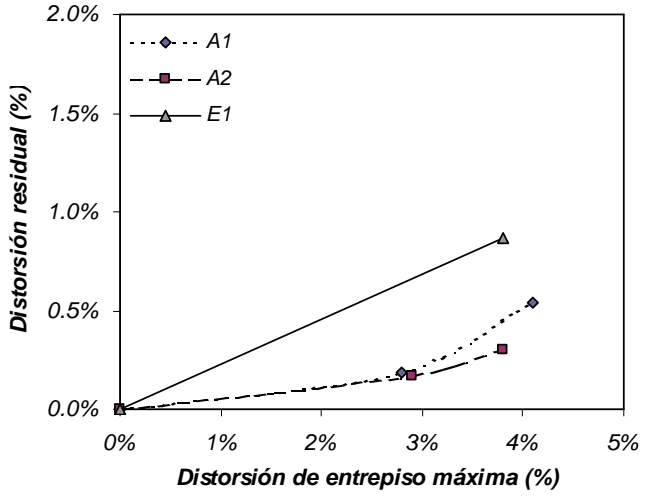

(b) Distorsión residual y de entrepiso máxima.

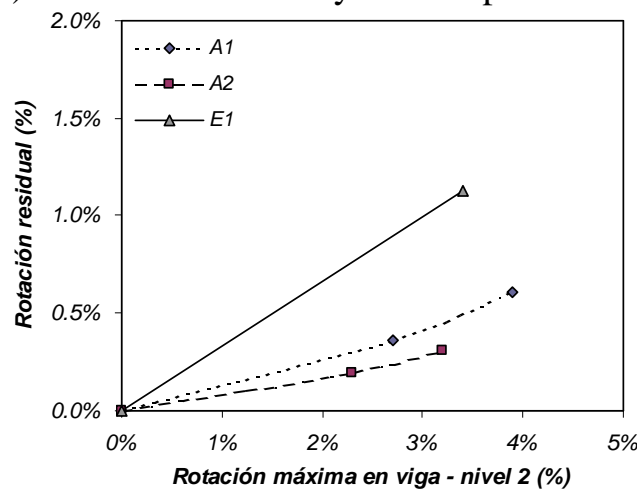

(c) Rotaciones residuales y máximas en secciones críticas de vigas y columnas de marcos

Figura 15. Deformaciones máximas y deformaciones residuales medidas en los especímenes

La falla del sistema en el espécimen E1 se manifestó en las articulaciones plásticas de los fusibles del marco, las que de acuerdo con las mediciones obtenidas con potenciómetros (figura 16a), presentaron ductilidades de rotación máximas iguales a 4.9 en vigas y 5.2 en columnas, y en la parte inferior del muro, donde la mayor cantidad de grietas se observó hasta una altura aproximada de 2.5 veces la longitud del muro (figura 16a). En la base del muro de este espécimen se obtuvieron grietas de hasta $0.5 \mathrm{~mm}$ de espesor (Sánchez, 2008).

La falla del sistema en los especímenes A1 y A2, se concentró en la base del muro autocentrado y en las articulaciones plásticas en los fusibles del marco, como se esperaba. Las ductilidades de rotación máximas obtenidas en el sismo de intensidad máxima aplicada en el espécimen A1 fueron iguales a 6.3 en vigas y 7.1 en columnas, los valores correspondientes en el espécimen A2 fueron iguales a 6.6 y 7.1, respectivamente. En la figura $16 \mathrm{~b}$ se muestran detalles del estado final de la base del muro del espécimen A1 después del sismo de intensidad máxima aplicada, en la base del muro se presentó el desprendimiento del recubrimiento y el aplastamiento del concreto confinado en un ancho de aproximadamente $0.05 \mathrm{~m}$. De manera similar, en la figura $16 \mathrm{c}$ se observan los daños producidos en la base de uno de los muros del espécimen A2 después del ensaye con intensidad máxima. Como se aprecia, de manera semejante a lo observado en el espécimen A1, se desprendió el recubrimiento y se aplastó el concreto confinado en un ancho con un valor cercano al observado en el espécimen A1. 

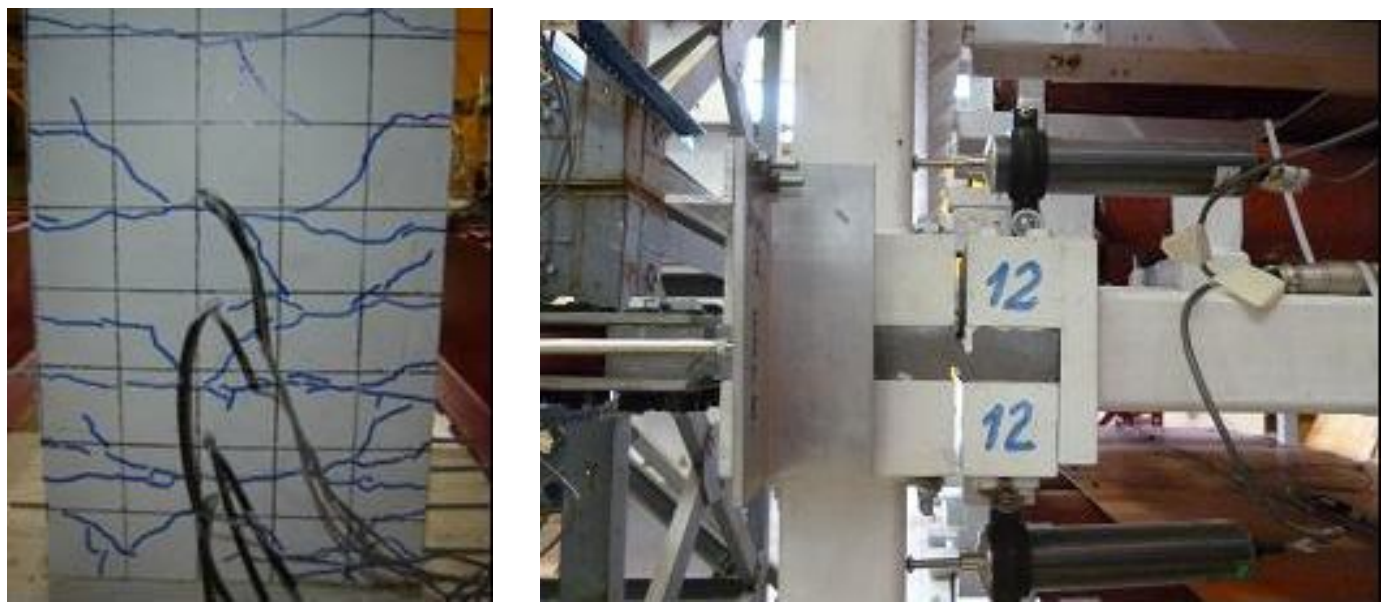

(a) Espécimen E1, daños en base de muro (grietas remarcadas) y rotaciones en fusibles después del ensaye de intensidad de diseño

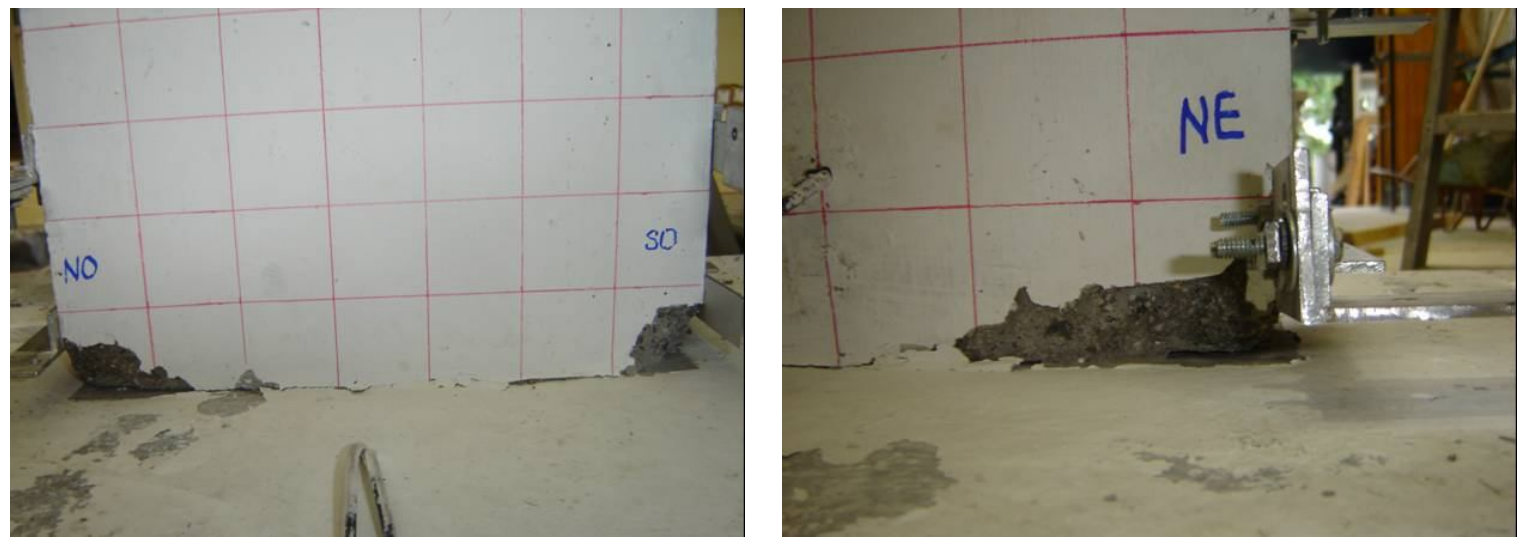

(b) Espécimen A1, daños en base de muro después del ensaye de intensidad máxima aplicada
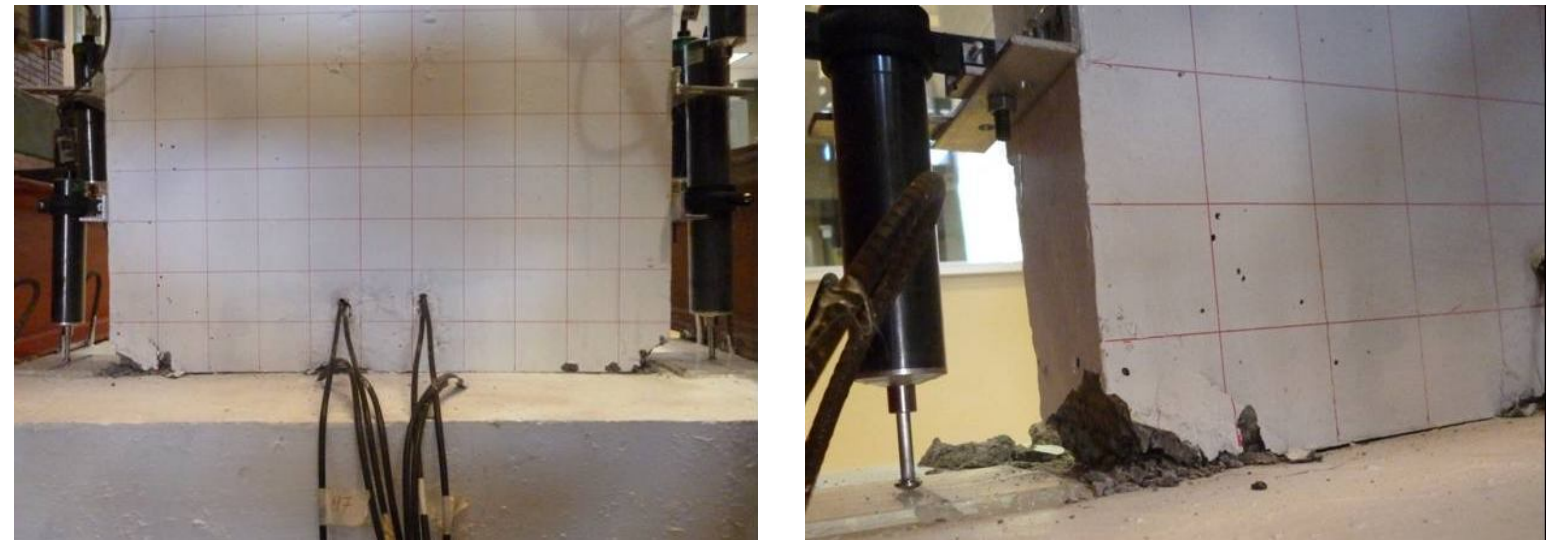

(c) Espécimen A2, daños en base de muro después del ensaye de intensidad máxima aplicada.

Figura 16. Forma de falla en los especímenes 


\section{ANÁLISIS ESTRUCTURAL}

Se prepararon modelos para la realización de los análisis estructurales elástico e inelástico no lineal de las estructuras ensayadas. Para el espécimen con muro convencional, el modelo de análisis fue preparado con base en criterios para el análisis de estructuras muro-marco calibrados con ensayes realizados en investigaciones precedentes (Rodríguez et al., 2006; Sánchez, 2008). Para los especímenes con marcos y muros autocentrados, la calibración del modelo se realizó con las mediciones efectuadas en los especímenes A1 y A2.

\section{Análisis de los especímenes}

Los especímenes fueron analizados con el programa Ruaumoko (Carr, 2010). Debido a la simetría del espécimen y a la ausencia de acciones sísmicas perpendiculares al sentido del ensaye, se empleó un modelo bidimensional, como se muestra en la figura 17 para el espécimen A1. Se concentró la masa en cada nivel y se ligaron los desplazamientos horizontales a dicho nudo para representar la acción de diafragma rígido. Se consideró que las bases del marco se encontraban empotradas, mientras que para la base de los muros autocentrados se incluyeron dos resortes rotacionales con el fin de representar la posibilidad de rotación de esta base. En el caso del espécimen con muro convencional, su base se consideró empotrada.

Para los modelos de análisis con el programa Ruaumoko (Carr, 2010), en los fusibles se emplearon elementos tipo spring de cuatro nudos con segmentos rígidos para representar los bloques macizos que sirvieron para fijar los fusibles en la unión viga-columna y en la base de las columnas. Para las vigas y las columnas se utilizaron elementos tipo frame de dos nudos con comportamiento elástico. Las bielas que unieron el muro y el marco fueron representadas mediante elementos tipo spring de dos nudos, con la rigidez axial equivalente de la armadura de conexión, figura 17. Se empleó la regla de histéresis de Takeda-Modificado (Carr, 2010) para los elementos de concreto reforzado, con los parámetros $\alpha=0.5$ y $\beta$ $=0$, y la regla de histéresis de Dodd-Restrepo (Dodd y Restrepo, 1995) para los fusibles de acero en el marco.

En la base de los muros autocentrados se incluyeron dos resortes rotacionales, mediante un elemento tipo ring-spring y otro elemento tipo bilineal-elástico (figura 18), para modelar las características del autocentrado con las barras disipadoras, de modo que tuviera las propiedades calculadas de acuerdo con los criterios indicados en la sección siguiente para los muros autocentrados. El cuerpo del muro fue modelado como un elemento tipo frame.

Para modelar el muro convencional del espécimen E1, se definieron modelos bilineales aproximados a partir de los diagramas momento-curvatura de las secciones del muro obtenidos con el programa BIAX (Wallace, 1989). Con estos diagramas, mediante una aproximación bilineal, se obtuvieron las inercias efectivas de las dos secciones típicas del muro, anteriormente comentadas, que correspondieron a $54 \%$ de la inercia de la sección bruta para el muro en sus tres primeros pisos y $40 \%$ para los pisos 4 y 5 (Salinas et al., 2011). La longitud de la articulación plástica en la base del muro fue calculada empleando la propuesta de Paulay y Priestley (1992), según la cual esta longitud depende del esfuerzo de fluencia del acero de la barra longitudinal, $f_{y}$ (en MPa), el diámetro de la barra longitudinal, $d_{b L}$, y la longitud del claro de cortante, $L$, con las longitudes dadas en milímetros:

$$
L_{p}=0.08 L+0.022 f_{y} d_{b L} \quad \geq 0.044 f_{y} d_{b L}
$$


Se debe mencionar que la ec. 5 originalmente fue propuesta para secciones típicas de vigas y columnas; sin embargo, la longitud de articulación plástica que se obtiene con esta expresión cae dentro del intervalo $0.3<L_{p} / L_{w}<8$ propuesto por Paulay y Priestley (1992) para la longitud de articulación plástica en muros estructurales de concreto, donde $L_{w}$ es el ancho del muro.

La tabla 2 presenta las propiedades del muro del espécimen E1 en las dos secciones típicas que se definieron considerando el cambio del acero de refuerzo longitudinal en elevación del muro (figura 4), tales como la inercia efectiva de la sección en el tramo inicial, $I_{e f}$, el factor de la pendiente post-fluencia, $r$, el momento de fluencia, $M_{y}$, y el momento último, $M_{u}$.

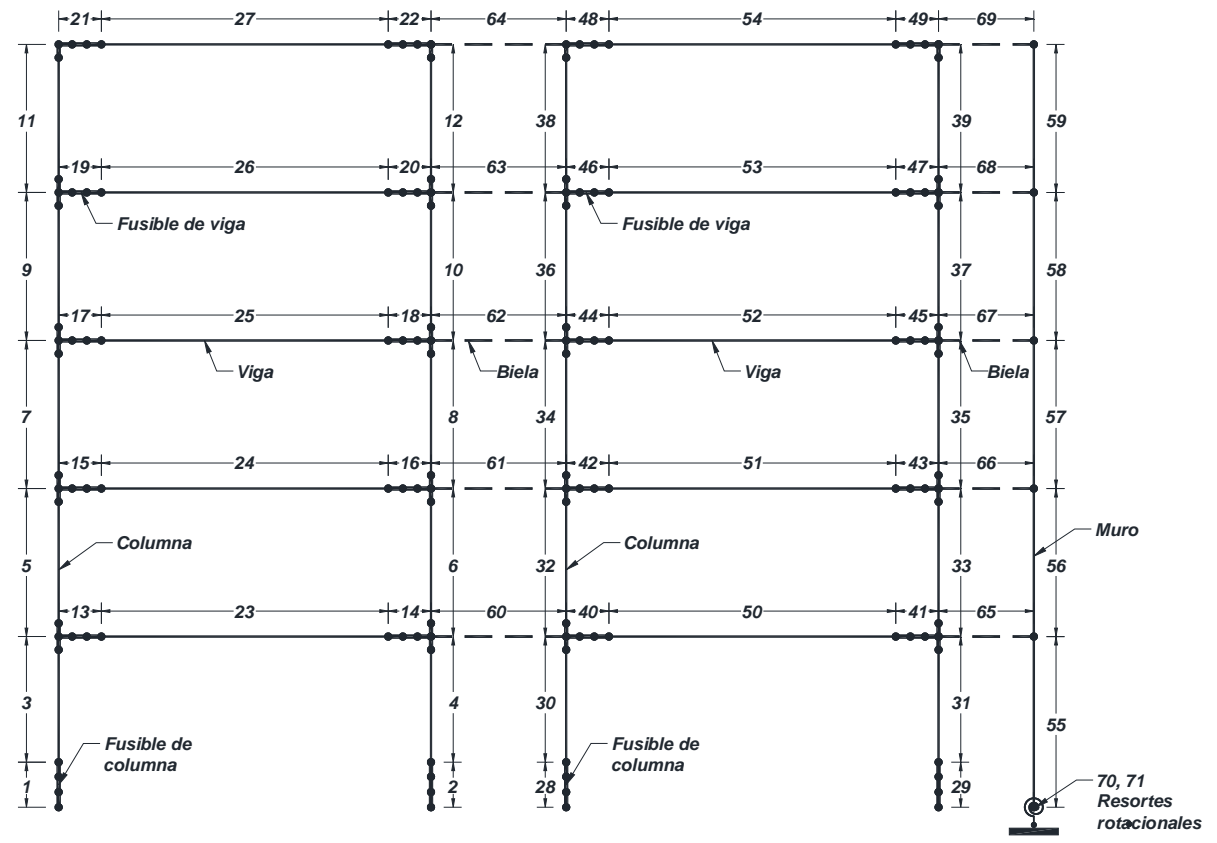

Figura 17. Modelo de análisis del espécimen A1



(a) Ring-spring

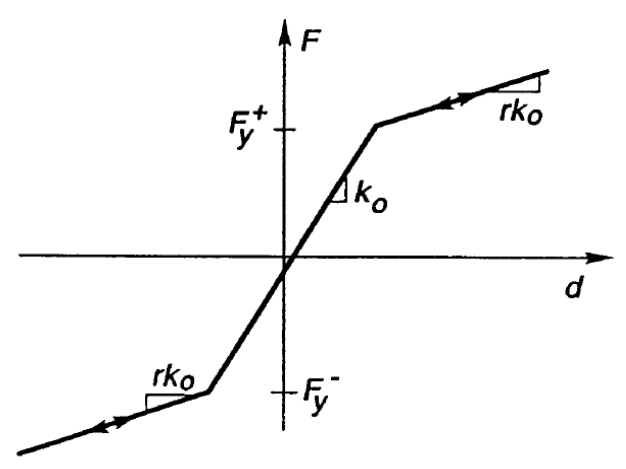

(b) Bilineal elástico

Figura 18. Reglas de histéresis empleadas en los resortes de la base del muro autocentrado (Carr, 2010) 
Tabla 2. Propiedades del muro, espécimen E1

\begin{tabular}{ccccc}
\hline Pisos & $\begin{array}{c}I_{\text {ef }} \\
\left(\mathrm{m}^{4}\right)\end{array}$ & $\begin{array}{c}r \\
\text { (pendiente post- } \\
\text { fluencia) }\end{array}$ & $\begin{array}{c}M_{y} \\
(\mathrm{kN}-\mathrm{m})\end{array}$ & $\begin{array}{c}M_{u} \\
(\mathrm{kN}-\mathrm{m})\end{array}$ \\
\hline 1 a 3 & $0.567 \times 10^{-4}$ & 0.024 & 20.6 & 25.4 \\
4 y 5 & $0.418 \times 10^{-4}$ & 0.020 & 15.2 & 18.4 \\
\hline
\end{tabular}

Las propiedades de rigidez y resistencia de los fusibles para describir la regla de histéresis de DoddRestrepo (1995) que emplea el programa Ruaumoko, se obtuvieron empleando el procedimiento descrito en Rodríguez et al. (2006). Este procedimiento emplea las inercias efectivas para la sección transversal de los fusibles. La tabla 3 resume las propiedades de los fusibles empleados en los modelos de análisis de los especímenes E1, A1 y A2, tales como la rigidez efectiva la flexión, $K_{\theta}$, la rotación al inicio del endurecimiento, $\theta_{s h}$, la rotación última, $\theta_{u}$, el momento plástico de la sección, $M_{p}$, y el momento último, $M_{u}$. La rigidez efectiva a la flexión está definida con la expresión:

$$
K_{\theta}=\frac{E_{s} I_{e f}}{L_{f}}
$$

donde $E_{s}$ es el módulo de elasticidad del acero del fusible, $I_{e f}$ es la inercia efectiva del fusible y $L_{f}$ es la longitud del fusible. Rodríguez et al. (2006) y Blandón (2006) realizaron ensayes de elementos estructurales con fusibles y obtuvieron valores de rigideces $K_{\theta}$ experimentales para definir la inercia efectiva. Se empleó la siguiente expresión que se aproxima razonablemente a los valores experimentales descritos en los trabajos citados (Salinas, 2013):

$$
K_{\theta}=\frac{5}{8} \frac{M_{u}}{\theta_{s h}}
$$

Con las ecs. 6 y 7, se obtiene la siguiente expresión para la inercia efectiva, $I_{e f}$ :

$$
I_{e f}=\frac{15}{16} \frac{f_{u}}{E_{s} \varepsilon_{s h}} I_{g}
$$

donde $f_{u}$ es el esfuerzo de rotura del acero, $I_{g}$ es el momento de inercia de la sección bruta del fusible y $\varepsilon_{s h}$ es la deformación del acero al inicio del endurecimiento. Con esta expresión, las inercias efectivas correspondieron a $25 \%, 30 \%$ y $22 \%$ de la inercia de la sección bruta para los especímenes E1, A1 y A2, respectivamente.

En los análisis no lineales se consideraron los efectos P-delta y la fracción de amortiguamiento crítico para cada modo fue considerada constante. Los valores de la fracción de amortiguamiento crítico fueron tomados a partir de las mediciones experimentales de la respuesta elástica de los especímenes en los ensayes de vibración libre, que permitieron obtener un intervalo de valores para la fracción de amortiguamiento crítico a considerar en los modelos de análisis. De esta manera, la fracción de amortiguamiento crítico en el modelo del espécimen E1 fue igual a $2 \%$ en todos los modos, en el modelo del espécimen A1 fue igual a 1.5\% para el primer modo de vibración y $3 \%$ para los modos superiores, mientras que en el modelo del espécimen A2 fue igual a 3\% en todos los modos. Para la integración de la ecuación dinámica de equilibrio se empleó el método de aceleración constante de Newmark y un intervalo 
de integración de $0.0001 \mathrm{~s}$, con el que se verificó la convergencia en el cálculo de desplazamientos y aceleraciones.

Tabla 3. Propiedades de los fusibles de los marcos

\begin{tabular}{ccccccc}
\hline \multirow{2}{*}{ Espécimen } & Ubicación & $\begin{array}{c}K_{\theta} \\
(\mathrm{kN}-\mathrm{m} / \mathrm{rad})\end{array}$ & $\begin{array}{c}\theta_{s h} \\
(\mathrm{rad})\end{array}$ & $\begin{array}{c}\theta_{u} \\
(\mathrm{rad})\end{array}$ & $\begin{array}{c}M_{p} \\
(\mathrm{kN}-\mathrm{m})\end{array}$ & $\begin{array}{c}M_{u} \\
(\mathrm{kN}-\mathrm{m})\end{array}$ \\
\hline \multirow{2}{*}{ E1 } & Extremo-viga & 197.6 & 0.0084 & 0.1262 & 1.43 & 2.15 \\
& Base-columna & 246.7 & 0.0078 & 0.1171 & 1.66 & 2.49 \\
\hline \multirow{2}{*}{$\mathrm{A} 1$} & Extremo-viga & 237.1 & 0.0071 & 0.1231 & 1.46 & 2.14 \\
& Base-columna & 296.1 & 0.0066 & 0.1143 & 1.69 & 2.49 \\
\hline \multirow{2}{*}{ A2 } & Extremo-viga & 173.8 & 0.0076 & 0.1385 & 1.25 & 2.13 \\
& Base-columna & 217.1 & 0.0071 & 0.1286 & 1.45 & 2.48 \\
\hline
\end{tabular}

\section{Modelo de análisis para la base del muro autocentrado}

Se calculó la curva momento-rotación de la base de los muros con base en las consideraciones del documento ACI-ITG-5.2-09 (2009), empleando las características geométricas de la sección transversal y las propiedades mecánicas de los materiales usados. Se distinguieron seis estados principales para definir la curva: (a) decompresión o levantamiento inminente del muro; (b) fluencia en el disipador más tensionado, al que corresponden los valores de momento y rotación $M_{y}$ y $\theta_{y}$, respectivamente; (c) fluencia en los dos disipadores; (d) inicio del endurecimiento por deformación en un disipador; (e) inicio del endurecimiento en los dos disipadores; y (f) rotura del disipador más tensionado o fluencia del tendón más tensionado (lo que ocurra primero según el criterio de diseño), a este estado corresponden los valores de momento y rotación $M_{u}$ y $\theta_{u}$, respectivamente. Estos estados para la base de los muros de los especímenes A1 y A2 se muestran en la figura 19.

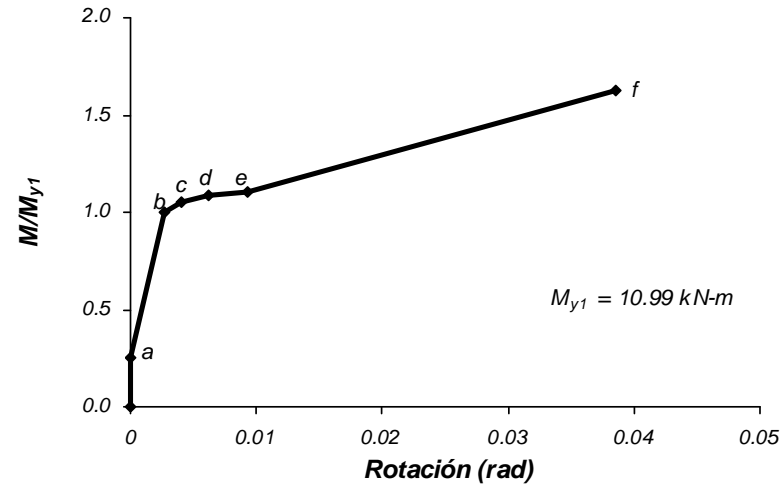

(a) Espécimen A1

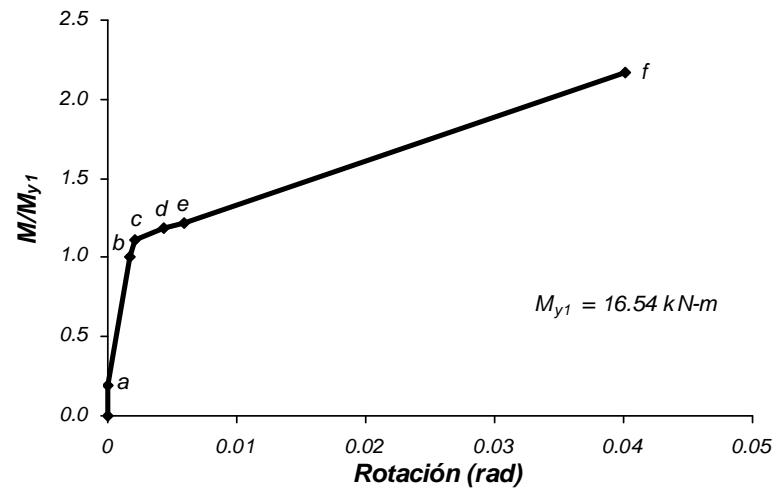

(b) Espécimen A2

Figura 19. Diagrama $M-\theta$ para la base de los muros autocentrados 
Para el cálculo de las rotaciones, la consideración de una longitud efectiva de la barra disipadora, $L_{d}$, fue importante para añadir a la longitud libre del disipador, $L_{s}$ (indicada para los especímenes A1 y A2 en las figuras 5 c y 6 c, respectivamente), una longitud de barra dentro de la zona adherida en el concreto. Esta consideración se emplea en el caso de disipadores formados por barras corrugadas de acero en la zona adherida al concreto y toma en cuenta el mecanismo de transferencia de las fuerzas por adherencia en esta zona. Este es el caso de los disipadores tipo "dog bone" empleados en los muros, que están formados por barras corrugadas en la zona adherida al concreto. El ACI-ITG-5.2-09 (ACI-ITG 5, 2009) recomienda el uso de la expresión $L_{d}=L_{s}+\alpha d_{b}$, donde $L_{s}$ es la longitud libre de la barra disipadora, $d_{b}$ el diámetro y $\alpha$ varía entre 2.0 y 5.5. En este estudio, para el cálculo de $L_{d}$ se empleó esta recomendación y se adoptó el valor de 5.5 .

Para generar el modelo de análisis para la base del muro autocentrado se emplearon los criterios siguientes:

a) Una primera rigidez rotacional de la base del muro, la que se calcula considerando la fluencia del disipador más tensionado (fig. 20).

b) Para considerar la disminución progresiva de la rigidez de la base del muro autocentrado, se estimó una segunda rigidez rotacional tomando en cuenta la intersección de la línea que une el punto de fluencia del disipador más tensionado y el punto en la última pendiente asociada a una línea horizontal trazada para un valor de momento igual al $75 \%$ del momento calculado en el estado último (figura 20). A este valor de momento le corresponde una rotación $\theta_{y R}$. Considerando una variación lineal entre el punto de momento de fluencia del disipador más tensionado y el punto de momento último, la rotación $\theta_{y R}$ se define con la expresión:

$\theta_{y R}=\left[\frac{0.25}{1-\frac{M_{y}}{M_{u}}}\right] \theta_{y}+\left[1-\frac{0.25}{1-\frac{M_{y}}{M_{u}}}\right] \theta_{u}$

Se propone una transición entre la rigidez inicial y la rigidez en la siguiente etapa de comportamiento lo que se ilustra en la figura 20.

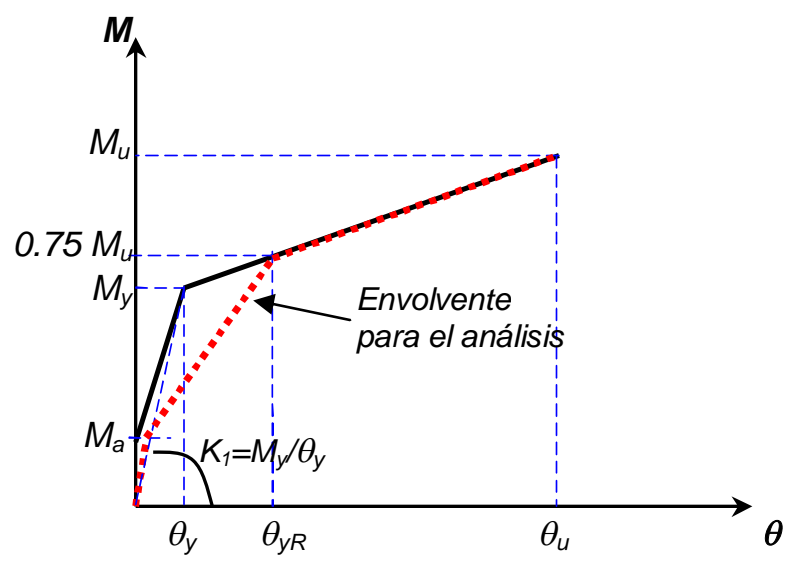

Figura 20. Envolvente del modelo propuesto para la base del muro autocentrado 
c) Una tercera rigidez rotacional es calculada con los valores de momento y rotación en el estado último y en el estado en que el momento es igual al $75 \%$ del momento calculado en el estado último (figura 20).

La envolvente trilineal generada (figura 20) se puede desarrollar con dos resortes rotacionales, uno de ellos con un elemento tipo ring-spring y el otro con un elemento tipo bilineal-elástico. El cuerpo del muro fue modelado como un elemento tipo frame con la inercia de la sección igual a la inercia de la sección bruta, ya que se espera un comportamiento principalmente elástico en el muro. En la tabla 4 se muestran los parámetros que definieron el modelo de resortes en la base de los muros autocentrados de los especímenes A1 y A2, respectivamente.

Tabla 4. Propiedades de la base de los muros autocentrados

\begin{tabular}{|c|c|c|c|c|c|c|}
\hline Espécimen & Resorte & $\begin{array}{c}K_{\theta} \\
(\mathrm{kN}-\mathrm{m} / \mathrm{rad})\end{array}$ & $\begin{array}{c}r \\
\text { (pendiente } \\
\text { post- } \\
\text { fluencia) }\end{array}$ & $\begin{array}{c}M_{y} \\
(\mathrm{kN}-\mathrm{m})\end{array}$ & $r_{\text {steep }}$ & $r_{\text {lower }}$ \\
\hline A1 & $\begin{array}{c}\text { Ring-spring } \\
\text { Bilineal }\end{array}$ & $\begin{array}{c}756.8 \\
3416.8\end{array}$ & $\begin{array}{c}0.247 \\
0\end{array}$ & 2.20 & 1.606 & 0.060 \\
\hline $\mathrm{A} 2$ & $\begin{array}{c}\text { Ring-spring } \\
\text { Bilineal }\end{array}$ & $\begin{array}{l}1353.8 \\
8397.4\end{array}$ & $\begin{array}{c}0.421 \\
0\end{array}$ & 2.72 & 2.451 & 0.116 \\
\hline
\end{tabular}

\section{COMPARACIÓN DE RESULTADOS ANALÍTICOS Y EXPERIMENTALES}

\section{Periodos de vibración y amortiguamientos modales}

La tabla 5 resume los periodos de vibración obtenidos con las mediciones experimentales de los especímenes en el ensaye de baja intensidad y aquellos calculados con los modelos de análisis. Estos periodos $\left(\mathrm{T}_{1}, \mathrm{~T}_{2}\right)$, para la respuesta elástica (sismo de baja intensidad) se han mostrado en la figura 13 . Como se ha mencionado, no fue posible obtener el segundo modo de vibración del espécimen A2. En la figura 21 se muestran las formas de las contribuciones modales, $\Gamma_{m i}$, definidas para un modo i-ésimo como el producto del factor de participación modal $\Gamma_{i}$ y las amplitudes modales $\phi_{i}$, calculadas con los modelos analíticos (modo i-c) y las obtenidas con las mediciones experimentales (modo i-e), en los tres especímenes, observándose una concordancia aceptable. Las amplitudes modales a partir de la información experimental fueron obtenidas aplicando el procedimiento descrito por Elgamal (2005). Los amortiguamientos modales fueron obtenidos mediante el procedimiento empleado por Rodríguez et al. (2006), con base en resultados experimentales a partir de la respuesta elástica de los especímenes en los ensayes de baja intensidad. Para el espécimen A1, los porcentajes de amortiguamiento calculados con los resultados experimentales fueron iguales a $1.5 \%$ y $2.0 \%$ para el primer y segundo modo, respectivamente. Para el espécimen A2, el porcentaje de amortiguamiento calculado con los resultados experimentales para el modo predominante fue igual a $4.0 \%$, este valor fue ligeramente mayor que el calculado con los resultados del ensaye de vibración libre, el cual fue igual a 3.0\%. Para el espécimen E1, los porcentajes de amortiguamiento calculados con los resultados experimentales (Sánchez, 2008) fueron iguales a 1.5\% y $3.0 \%$ para el primer y segundo modo, respectivamente.

La comparación de los resultados analíticos y experimentales que se presenta a continuación corresponde a los ensayes en mesa vibradora para los sismos de intensidad de diseño. Para los ensayes de intensidad máxima aplicada, la correlación obtenida entre resultados experimentales y calculados fue 
similar, por lo que para este nivel de intensidad sísmica las conclusiones presentadas en esta sección son semejantes.

Tabla 5. Periodos de vibración (s)

\begin{tabular}{cccc}
\hline Espécimen & & Modo 1 & Modo 2 \\
\hline \multirow{2}{*}{ E1 } & Medido & 0.41 & 0.09 \\
& Calculado & 0.41 & 0.10 \\
\hline \multirow{2}{*}{ A1 } & Medido & 0.38 & 0.08 \\
& Calculado & 0.37 & 0.06 \\
\hline \multirow{2}{*}{ A2 } & Medido & 0.26 & -- \\
& Calculado & 0.24 & 0.03 \\
\hline
\end{tabular}

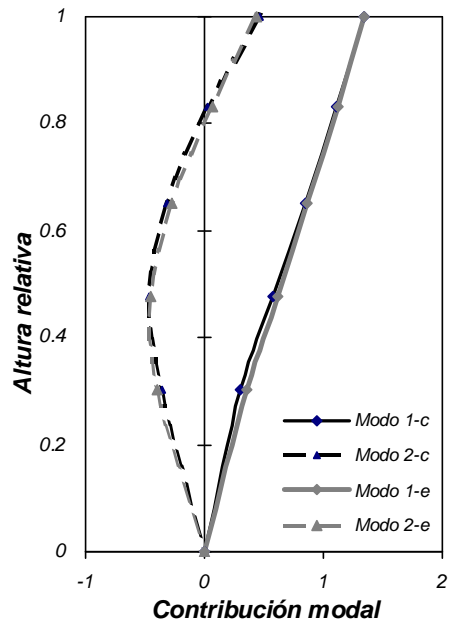

(a) Espécimen E1

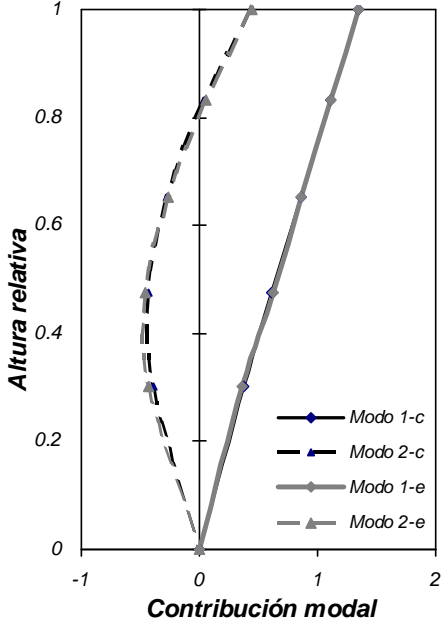

(b) Espécimen A1

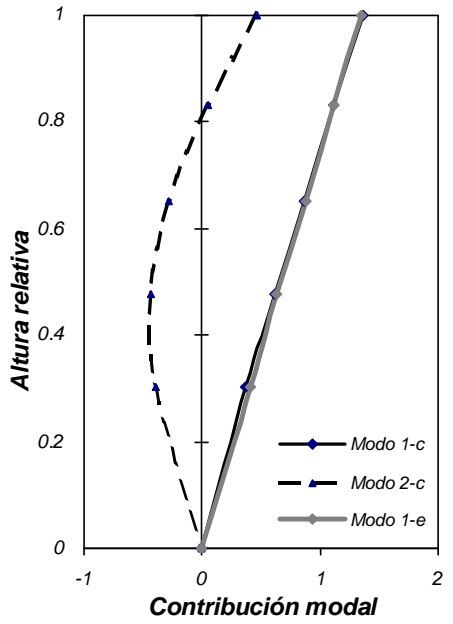

(c) Espécimen A2

Figura 21. Contribuciones modales experimentales y calculadas

\section{Sismo de intensidad de diseño}

Las figuras 22 a 24 permiten comparar diversos resultados experimentales y calculados empleando el programa Ruaumoko. En estas figuras, los resultados experimentales medidos se muestran en línea gruesa clara y los resultados calculados con el modelo de análisis se muestran en línea delgada oscura. Las figuras (a) permiten comparar las envolventes de desplazamiento de piso relativo a la base, $\Delta_{i}$, dividido entre la altura total del edificio, $H$, en función de la altura relativa de piso (cociente entre la altura total de piso y la altura total del edificio). Las figuras (b) presentan las envolventes de aceleraciones absolutas máximas de piso con la altura relativa de piso. Las figuras (c) permiten comparar las magnitudes del espectro de Fourier y el contenido de frecuencias de las aceleraciones en el piso 5. Las figuras (d) presentan los ciclos de histéresis de momento de volteo en función de la distorsión global (cociente entre el desplazamiento del último piso entre la altura total del espécimen).

La envolvente de desplazamientos del espécimen E1 (figura 22a) presenta un perfil ligeramente curvo, lo cual sugiere un comportamiento de flexión debido a la presencia del muro. Esta figura y la figura 
22b, que muestra la envolvente de aceleraciones absolutas máximas de piso, permite apreciar que la correlación entre los resultados medidos y calculados es aceptable, con la excepción de la aceleración del piso 5, que fue de hasta 2 veces la aceleración máxima de la base, alrededor de $60 \%$ mayor que la calculada con el modelo de análisis, debido a las razones expuestas en la sección "Evaluación de resultados". La figura 22c permite apreciar la semejanza entre los espectros de Fourier de las aceleraciones máximas medidas y calculadas en el piso 5 (donde se registran las máximas aceleraciones absolutas), lo que muestra la bondad de la predicción del contenido de frecuencias en el intervalo inelástico. En los ciclos de histéresis del momento de volteo versus distorsión global (figura 22d) se observa que el modelo analítico sobreestima la capacidad de disipación de energía inelástica comparada con la de la respuesta experimental; las diferencias observadas en las magnitudes del momento de volteo entre los resultados medidos y calculados están asociadas a la anomalía detectada en las aceleraciones del piso 5 del espécimen por las razones comentadas en una sección anterior.

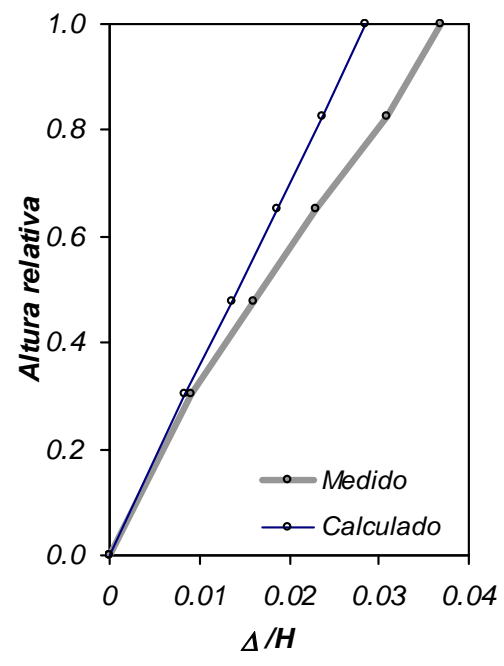

(a) Desplazamientos relativos a la base

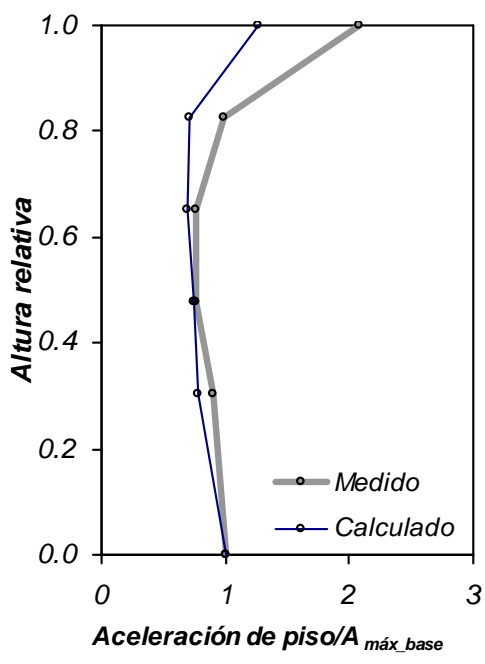

(b) Aceleraciones absolutas

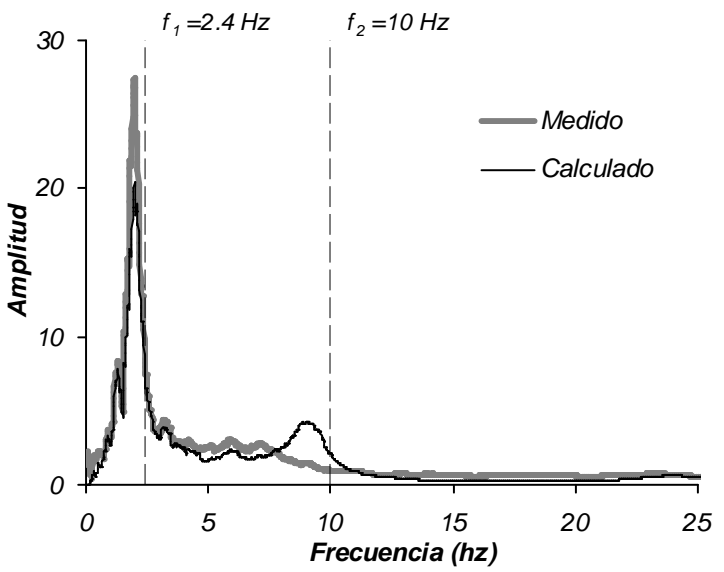

(c) Espectro de Fourier

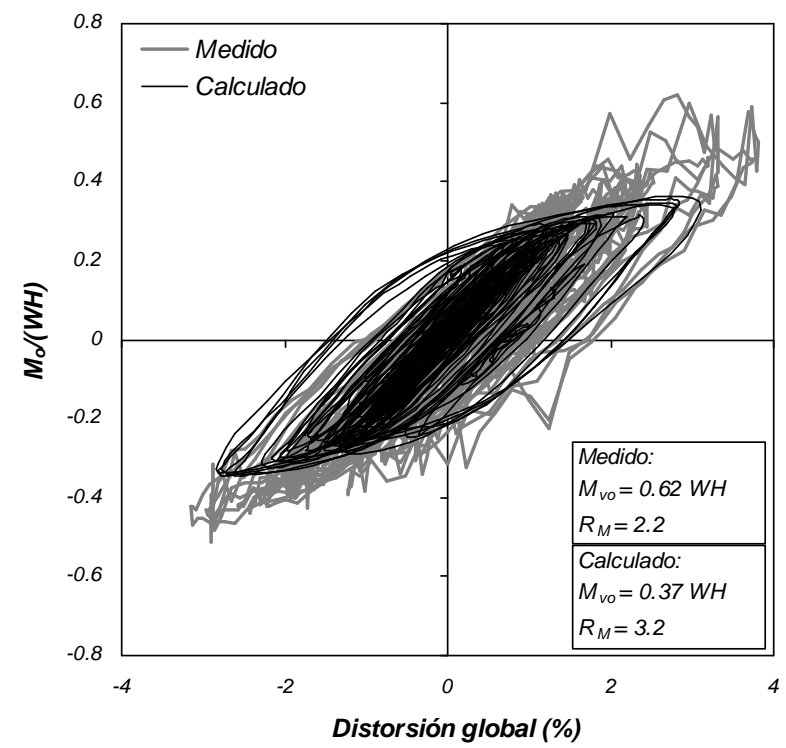

(d) Respuesta histerética global

Figura 22. Espécimen E1, respuesta ante el sismo de alta intensidad 
En el espécimen A1, la forma de la envolvente de desplazamientos (figura 23a) presenta un perfil prácticamente lineal, lo cual refleja el predominio del muro en el comportamiento del sistema total. Esta figura y la figura $23 \mathrm{~b}$, que muestra la envolvente de aceleraciones absolutas máximas de piso, permite apreciar que la correlación entre los resultados medidos y calculados es aceptable. La semejanza entre los espectros de Fourier (figura 23c), así como entre los ciclos de histéresis del momento de volteo versus distorsión global (figura 23d) permite apreciar la bondad de la predicción del contenido de frecuencias en el intervalo inelástico con el modelo propuesto. En la figura $23 \mathrm{~d}$ se aprecia que los valores máximos de distorsiones de entrepiso y momentos de volteo calculados fueron del orden del $85 \%$ de los valores encontrados experimentalmente.

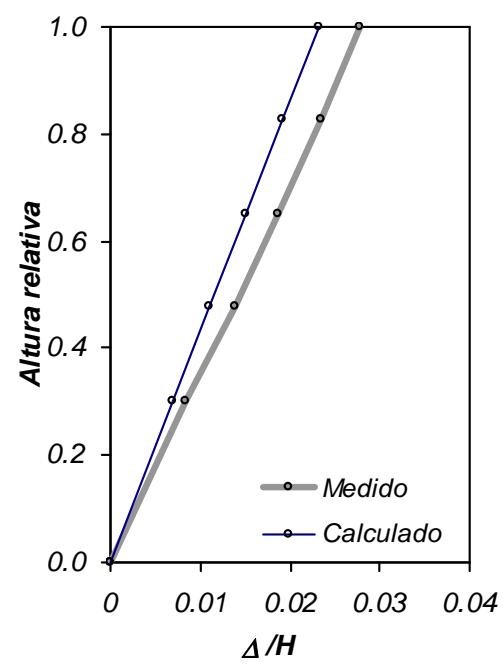

(a) Desplazamientos relativos a la base.

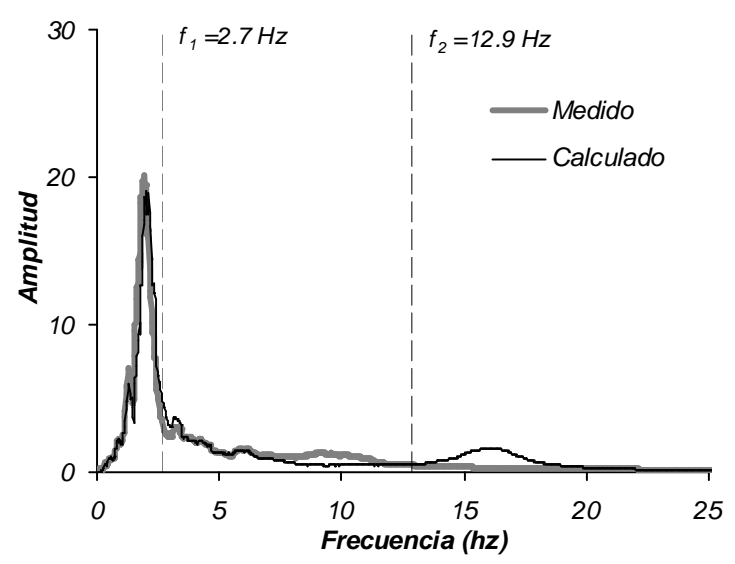

(c) Espectro de Fourier.

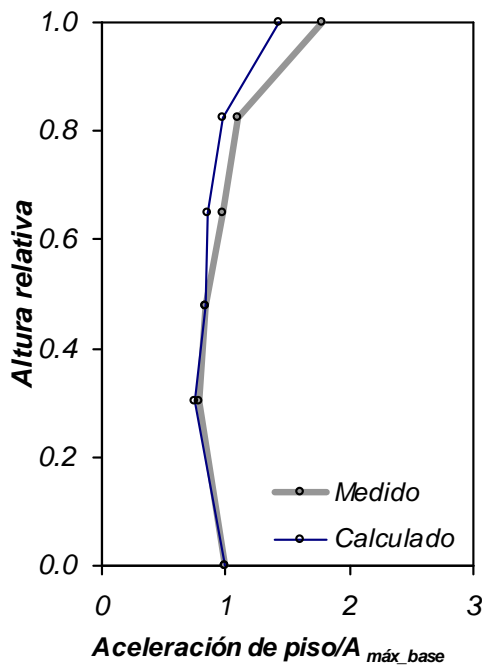

(b) Aceleraciones absolutas

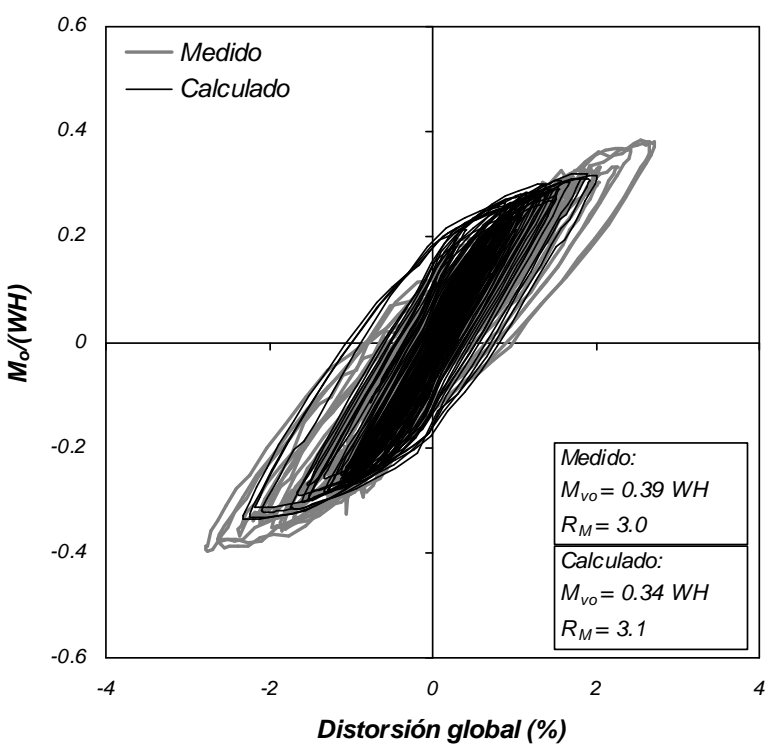

(d) Respuesta histerética global.

Figura 23. Espécimen A1, respuesta ante el sismo de alta intensidad 
En el espécimen A2, la envolvente de desplazamientos (figura 24a) presenta también un perfil prácticamente lineal, lo cual refleja el predominio del muro en el comportamiento del sistema. Las envolventes de las figuras $24 \mathrm{a}$ y $24 \mathrm{~b}$ permiten apreciar que la correlación entre los resultados medidos y calculados es aceptable. Del mismo modo que lo observado en el espécimen A1, la semejanza entre los espectros de Fourier (figura 24c), así como entre los ciclos de histéresis del momento de volteo versus distorsión global (figura 24d) permite apreciar la bondad de la predicción del contenido de frecuencias en el intervalo inelástico con el modelo propuesto. En la figura $24 \mathrm{~d}$ se aprecia que los valores máximos de distorsiones de entrepiso y momentos de volteo calculados fueron del orden del $80 \%$ y $94 \%$ de los valores medidos, respectivamente.

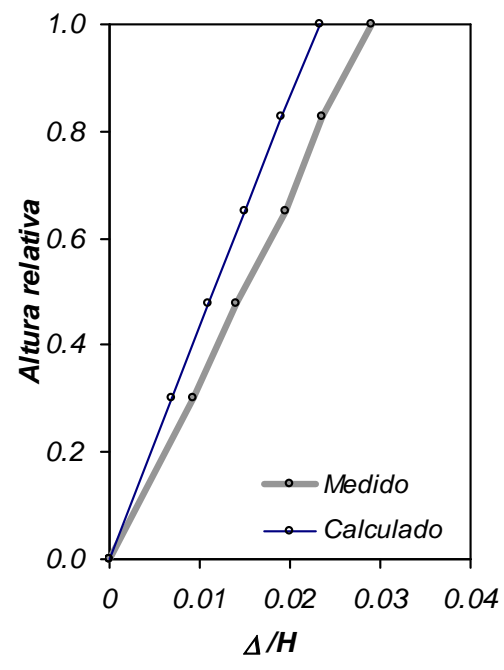

(a) Desplazamientos relativos a la base

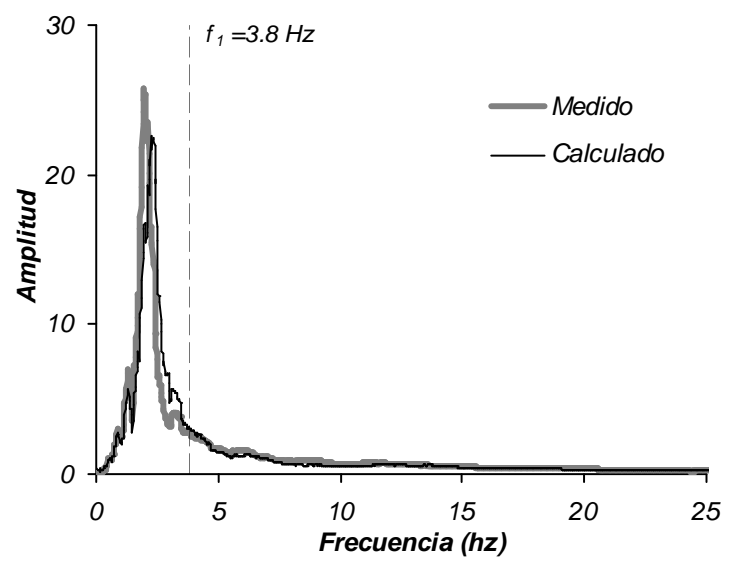

(c) Espectro de Fourier

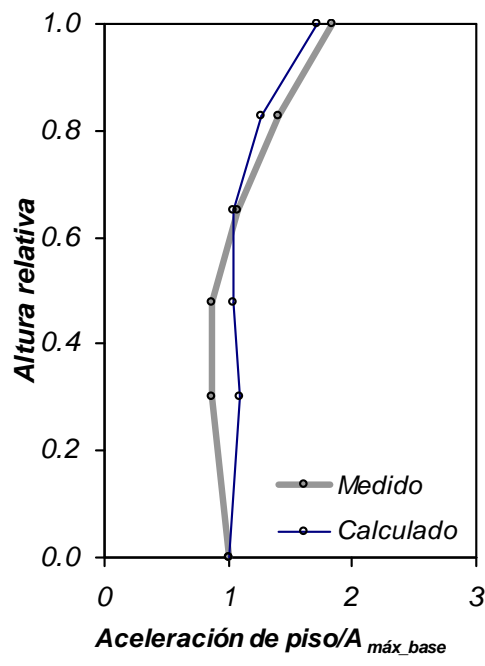

(b) Aceleraciones absolutas

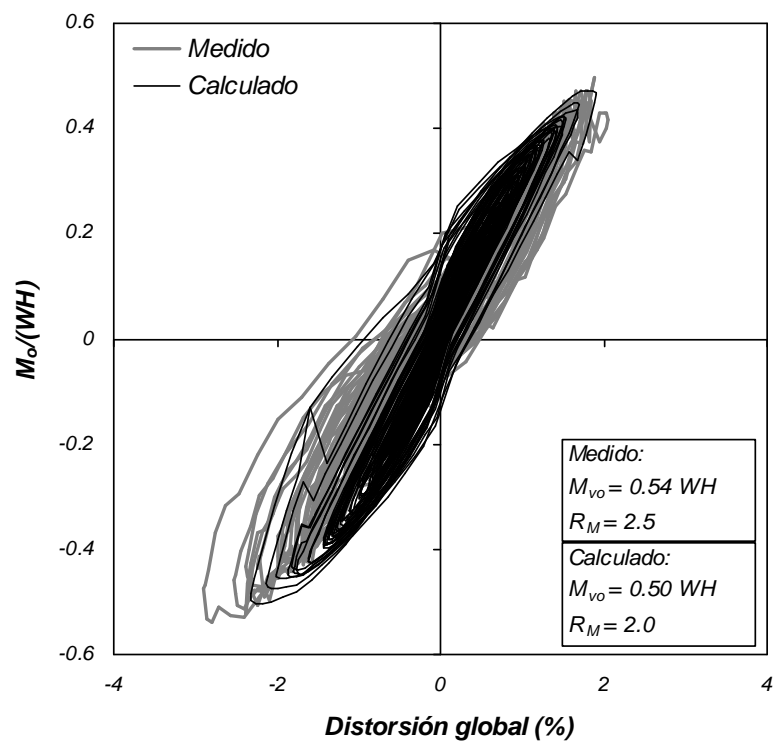

(d) Respuesta histerética global

Figura 24. Espécimen A2, respuesta ante el sismo de alta intensidad

Se calcularon algunos parámetros globales de la respuesta de los modelos analíticos y de los especímenes E1, A1 y A2, con los resultados de los movimientos de baja intensidad y de alta intensidad, 
que se presentan en la tabla 6 . Los parámetros globales presentados en la tabla 6 son el cociente $R_{M}$, la relación entre el cortante máximo en la base y el peso total del edificio, $V / W$, la sobrerresistencia $\Omega_{o}$, y la relación entre los desplazamientos máximos en el piso superior considerando una respuesta inelástica y aquél obtenido conservando una respuesta elástica, $D_{\text {rin }} / D_{\text {rel }}$. El cortante máximo en la base, $V$, fue calculado como el máximo valor de la historia de cortantes basales, valuados como la suma de las fuerzas inerciales de cada piso, que son iguales a su vez al producto de la masa por la aceleración absoluta horizontal de cada piso.

Tabla 6. Parámetros globales de respuesta

\begin{tabular}{|c|c|c|c|c|c|}
\hline \multirow{2}{*}{ Espécimen } & \multirow{2}{*}{ Parámetro } & \multicolumn{2}{|c|}{$\begin{array}{c}\text { Movimiento de } \\
\text { intensidad de diseño }\end{array}$} & \multicolumn{2}{|c|}{$\begin{array}{c}\text { Movimiento de } \\
\text { intensidad máxima aplicada }\end{array}$} \\
\hline & & Calculado & Experimental & Calculado & Experimental \\
\hline \multirow{4}{*}{ E1 } & $R_{M}$ & 3.2 & 2.2 & -- & -- \\
\hline & $V / W$ & 0.53 & 0.77 & -- & -- \\
\hline & $\Omega_{o}$ & 2.0 & 3.3 & -- & -- \\
\hline & $D_{\text {rin }} / D_{\text {rel }}$ & 0.78 & 0.90 & -- & -- \\
\hline \multirow{4}{*}{ A1 } & $R_{M}$ & 3.1 & 3.0 & 4.3 & 4.4 \\
\hline & $V / W$ & 0.48 & 0.54 & 0.55 & 0.57 \\
\hline & $\Omega_{o}$ & 1.8 & 2.1 & 2.1 & 2.3 \\
\hline & $D_{r i n} / D_{r e l}$ & 0.71 & 0.78 & 0.71 & 0.69 \\
\hline \multirow{4}{*}{ A2 } & $R_{M}$ & 2.0 & 2.5 & 2.3 & 3.0 \\
\hline & $V / W$ & 0.70 & 0.70 & 0.76 & 0.76 \\
\hline & $\Omega_{o}$ & 2.7 & 2.9 & 2.9 & 3.0 \\
\hline & $D_{\text {rin }} / D_{\text {rel }}$ & 1.15 & 1.35 & 1.10 & 1.46 \\
\hline
\end{tabular}

En general, los resultados calculados presentan una concordancia aceptable con los resultados experimentales. La concordancia de los resultados experimentales con los valores calculados con el modelo de análisis empleado para los dos especímenes autocentrados con características diferentes, tanto en periodos como en participación de los muros en el sistema dual, han permitido validar el modelo propuesto para el sistema muro-marco con muros autocentrados, descrito en la sección "Análisis Estructural" de este trabajo.

La figura 25 muestra la envolvente de las aceleraciones de piso divididas entre la aceleración máxima de la base, para los especímenes en los movimientos de intensidad de diseño y de intensidad máxima aplicada, medidas y calculadas por dos procedimientos analíticos. Estos modelos fueron el análisis modal espectral empleado en la práctica, con la técnica de la raíz cuadrada de la suma de los cuadrados y la reducción de todas las contribuciones modales por el factor $R_{M}$ (RCSC), y el análisis modal espectral con la reducción de la contribución del primer modo (Primer Modo Reducido, PMR; Rodríguez et al., 2002). En general, se observa una mejor correlación entre los valores medidos y los calculados reduciendo solamente la contribución del primer modo. Un caso particular es el del espécimen A2, que presenta una correlación notoria entre los valores medidos y los calculados con los dos procedimientos analíticos, debido a que en este espécimen la respuesta capturada experimentalmente correspondió al modo fundamental de vibración. En general, es posible aplicar procedimientos como el del PMR para el cálculo de las aceleraciones máximas de piso de las estructuras muro-marco con muros autocentrados, de manera similar a su aplicación en estructuras convencionales. 


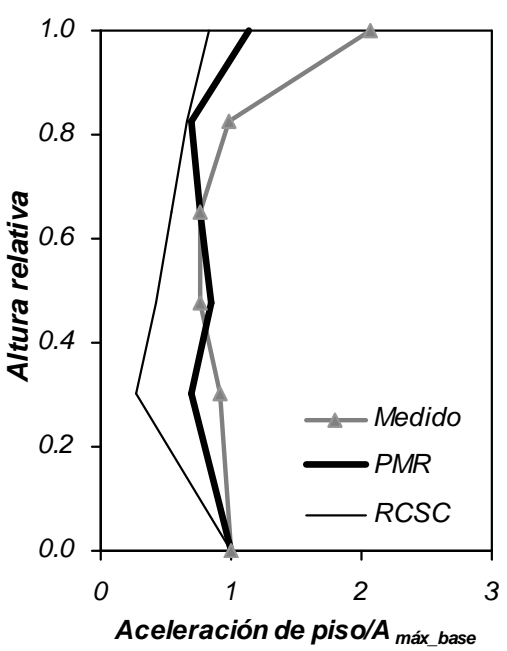

Espécimen E1

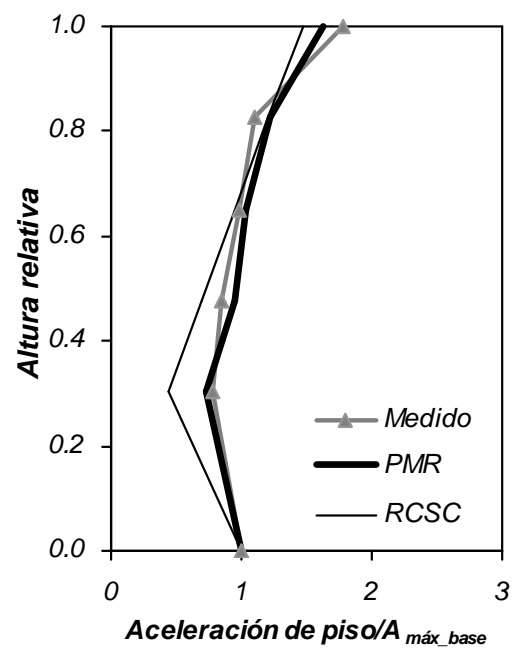

Espécimen A1

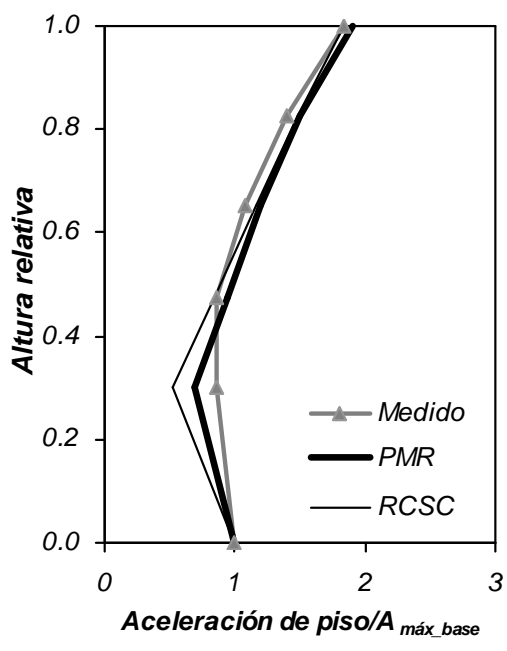

Espécimen A2

(a) Intensidad de diseño

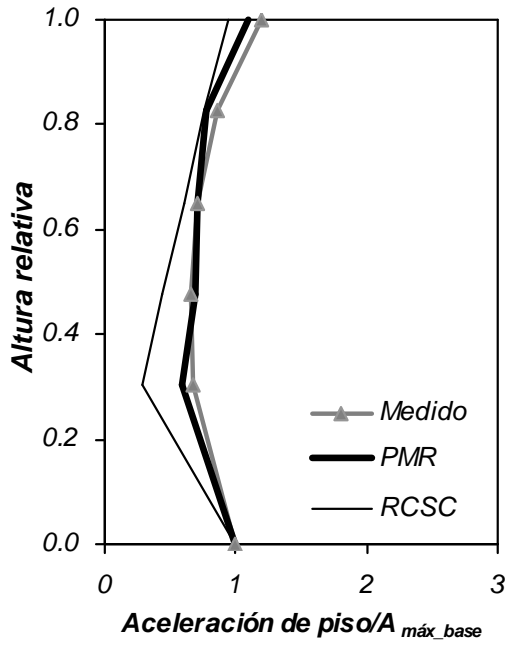

Espécimen A1

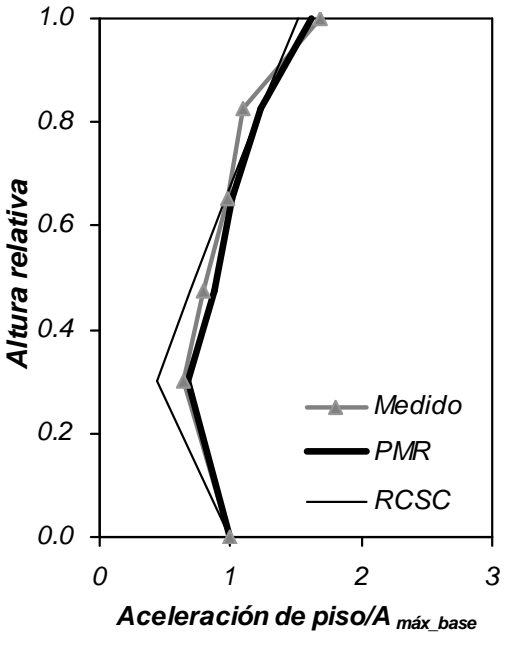

Espécimen A2

(b) Intensidad máxima aplicada.

Figura 25. Amplificación de aceleraciones de piso

\section{CONCLUSIONES}

En este trabajo se describen resultados de ensayes en mesa vibradora en tres especímenes miniatura de cinco pisos con un sistema estructural a base de marcos y muros, diseñados con un espectro de diseño sísmico similar y con parámetros de diseño sísmico empleados en estructuras duales convencionales. Dos especímenes tuvieron muros estructurales autocentrados de concreto presforzado con diferente participación en la respuesta sísmica total y un espécimen tuvo un muro estructural convencional de concreto reforzado. Los tres especímenes fueron sometidos a movimientos en la base de intensidad aproximada a la de diseño y los dos especímenes con muros autocentrados fueron sometidos adicionalmente a un movimiento en la base de intensidad mayor. Se prepararon modelos de análisis 
estructural para los especímenes, con los que se evaluó el desempeño sísmico de las estructuras ensayadas. Las conclusiones de este estudio son las siguientes:

1. Los especímenes ensayados con muros autocentrados tuvieron un desempeño sísmico comparable con el alcanzado en el espécimen con muro convencional, en términos de aceleraciones y desplazamientos máximos, sobrerresistencia y reducción por ductilidad. Este hecho fue más evidente en los especímenes A1 (autocentrado) y E1 (convencional), diseñados para tener una similar participación de los muros en términos del momento de volteo. Esto sugiere la posibilidad de establecer, para los sistemas muro-marco con muros autocentrados, criterios de diseño sísmico similares a los empleados en los sistemas con muros convencionales. Como se ha mencionado en este trabajo, una de las ventajas relevantes de los muros autocentrados, con respecto a los convencionales, es la reducción importante en las distorsiones residuales observadas al final del movimiento sísmico, por lo que su empleo en zonas sísmicas es recomendable.

2. El espécimen A2 (autocentrado) fue diseñado para tener una participación de muros, en términos del momento de volteo total de diseño, del orden de 2 veces mayor que la de los especímenes A1 y E1. El espécimen A2 presentó menores desplazamientos relativos del centro de masa de los pisos, pero presentó una mayor sobrerresistencia estructural, alrededor de 1.5 veces mayor que la sobrerresistencia calculada para los otros especímenes. En la asignación de un valor a la sobrerresistencia según el sistema estructural, con fines de diseño, se recomienda tomar en cuenta esta influencia, para evitar la subestimación de la resistencia probable de una estructura muro-marco con muros autocentrados y una evaluación inadecuada de su forma de falla.

3. En los dos especímenes con muros autocentrados, el cuerpo principal de los muros no presentó daños visibles, mientras que en los extremos de la base de los muros se observó el desprendimiento del concreto hasta distancias mayores que el recubrimiento. En el espécimen con muro convencional el cuerpo principal del muro presentó grietas, la mayor cantidad de las cuales se observó en la parte inferior del muro hasta una altura de 2.5 veces la longitud del muro.

4. La evaluación de la respuesta experimental de los especímenes mostró una mayor reducción de la respuesta sísmica en el modo fundamental de vibración, siendo menor la reducción en el segundo modo de vibración. Este hecho ha sido observado en trabajos anteriores de otros autores, y puede hacer posible la aplicación de procedimientos propuestos como el del Primer Modo Reducido para el cálculo de las aceleraciones de piso de las estructuras muro-marco con muros autocentrados, de manera similar a su aplicación en estructuras convencionales.

5. Las distorsiones relativas de entrepiso residuales medidas después del sismo de intensidad de diseño fueron mínimas en los especímenes con muros autocentrados, e iguales a $0.19 \%$ y $0.17 \%$, para los especímenes A1 y A2, respectivamente. Las distorsiones relativas de entrepiso residuales medidas para este sismo en el caso del espécimen E1 fueron bastante mayores que las medidas en los especímenes A1 y A2, del orden de 4.6 veces. Los sismos de intensidad máxima aplicados posteriormente a los especímenes con muros autocentrados implicaron un aumento de las distorsiones residuales; sin embargo, para este nivel de sismo, en el espécimen A1 la distorsión residual fue igual a $0.54 \%$ y en el espécimen A2 fue igual a $0.30 \%$. Se observó una reducción de las deformaciones residuales en las secciones críticas de los marcos de los especímenes con muros autocentrados, de similar proporción a la encontrada a nivel de las distorsiones residuales de la estructura total. 
6. Los modelos de análisis estructural para los especímenes con muros autocentrados presentaron resultados que tuvieron una correlación aceptable con los experimentales, en términos de las envolventes de aceleraciones absolutas y desplazamientos relativos y del contenido de frecuencias de la respuesta estructural.

7. Los resultados experimentales muestran que los sistemas muro-marco con muros autocentrados presentan en general un desempeño semejante al de un sistema muro-marco con muros convencionales, con la propiedad adicional de presentar menores desplazamientos residuales, por lo que este sistema estructural se podría emplear en edificios en los que se busque reducir los costos de reparación post-sismo.

\section{AGRADECIMIENTOS}

Se agradece el apoyo financiero de FINCYT (Programa de Ciencia y Tecnología) del Gobierno del Perú, al Latin American Scholarships Program for American Universities - LASPAU y la Universidad Nacional de Ingeniería - UNI, del Perú, para los estudios de doctorado e investigación del primero de los autores en la Universidad Nacional Autónoma de México - UNAM. Se reconoce el apoyo de UC MEXUS-CONACYT, a través del Grant Number CN-09318, que permitió financiar algunos de los ensayes en la mesa vibradora descritos en este estudio. Se agradece al Dr. José Restrepo de la Universidad de California, San Diego, por su acertada revisión crítica de este artículo, así como a los revisores del manuscrito por sus útiles comentarios al trabajo, que permitieron mejorar la calidad de éste.

\section{REFERENCIAS}

ACI Innovation Task Group 5 (2009), "Requirements for design of special unbonded post-tensioned precast shear wall satisfying ACI ITG-5.1 (ACI ITG-5.2-09) and Commentary", American Concrete Institute, Farmington Hills, MI, EE.UU.

Blandón, J. (2006), "Diseño sísmico de sistemas de piso en estructuras prefabricadas de concreto", Tesis de Doctorado, Facultad de Ingeniería, Universidad Nacional Autónoma de México, México.

Carr, A. (2010), "Ruaumoko", Computer Program Library, Departamento de Ingeniería Civil, Universidad de Canterbury. Christchurch, Nueva Zelanda.

Dodd, L. y Restrepo, J. (1995), "Model for predicting cyclic behaviour of reinforcing steel", Journal of Structural Engineering ASCE, Vol. 121, No. 3, pp. 433-445.

Elgamal, A. (2005). (http://webshaker.ucsd.edu/homework/CrossbowDataLogger.pdf)

Gaceta Oficial del Distrito Federal (2004), "Normas Técnicas Complementarias para Diseño y Construcción de Estructuras de Concreto". Reglamento de Construcciones del Distrito Federal. México DF.

Holden, T, Restrepo, J. y Mander, J. (2003), "Seismic performance of precast reinforced and prestressed concrete walls", Journal of Structural Engineering ASCE, Vol. 129, No.3, pp. 286-296.

Kao, G. (1998), "Design and shake-table test of a four-storey miniature structure built with replaceable plastic hinges", Tesis de Maestría, Departamento de Ingeniería Civil, Universidad de Canterbury. Christchurch, Nueva Zelanda.

Kurama, Y., Sause, R., Pessiki, S. y Lu, L.W. (1999), "Lateral load behavior and seismic design of unbonded post-tensioned precast concrete walls", ACI Structural Journal, Vol.96, No.4, pp. 622- 
633.

Martinelli, P., y Filippou, F., (2009), "Simulation of the shaking table test of a seven-story shear wall building", Earthquake Engineering and Structural Dynamics, Vol. 38, pp. 587-607.

Panagiotou, M., (2008), "Seismic design, testing, and analysis of reinforced concrete wall buildings", Tesis de Doctorado, Universidad de California, San Diego, USA, supervisada por J. Restrepo.

Pampanin, S., Priestley, M.J.N. y Sritharan, S. (2001), "Analytical modeling of the seismic behavior of precast concrete frames designed with ductile connections", Journal of Earthquake Engineering, Vol.5, No.3, pp. 329-367.

Paulay, T. y Priestley, M.J.N. (1992), Seismic design of reinforced concrete and masonry buildings. John Wiley \& Sons Inc., N.Y., EE.UU.

Pennucci, D., Calvi, G.M. y Sullivan, T.J. (2009), "Displacement-based design of precast walls with additional dampers", Journal of Earthquake Engineering, Vol.13 (S1), pp. 40-65.

Perez, F., Sause, R., y Pessiki S., (2007), "Analytical and experimental lateral load behavior of unbonded posttensioned precast concrete walls", Journal of Structural Engineering ASCE, Vol.133, No.11, pp. 1531-1540.

Priestley, M.J.N. (1996), "The PRESSS Program - Current status and proposed plans for phase III", Special report, PCI Journal, Vol.41, No.2, pp.22-40.

Priestley M.J.N. y Tao J.R., (1993), "Seismic response of precast prestressed concrete frames with partially debonded tendons", PCI Journal, Vol.38, No.1, pp 58-69.

Rahman, A., y Restrepo, J. (2001), "Earthquake resistant precast concrete buildings: Seismic performance of cantilever wall prestressed using unbonded tendons." Research Rep. No 2000-5, Departamento de Ingeniería Civil, Universidad de Canterbury, Christchurch, Nueva Zelanda.

Reglamento de Construcciones para los Municipios del Estado de Guerrero, (1989), Normas Técnicas Complementarias para Diseño por Sismo, Reporte Instituto de Ingeniería, UNAM.

Restrepo, J. y Rahman, A. (2007), "Seismic performance of self-centering structural walls incorporating energy dissipators", Journal of Structural Engineering ASCE, Vol.133, No.11, pp. 1560-1570.

Rodríguez, M.E., Restrepo, J. y Carr, A., (2002), "Earthquake induced horizontal floor accelerations in buildings", Earthquake Engineering and Structural Dynamics, Vol. 31, pp. 693-718.

Rodríguez, M.E., Restrepo, J. y Blandón, J. (2006), "Shaking table test of a four-story miniature steel building - model validation”, Earthquake Spectra, Vol. 22, No.3, pp. 755-780.

Rodríguez, M.E., Restrepo, J. y Blandón, J., (2007), "Seismic design forces for rigid floor diaphragms in precast concrete buildings structures", Journal of Structural Engineering ASCE, Vol. 133, No. 11, pp. 1604-1615.

Rodríguez, M.E. y Restrepo, J. (2012), "Práctica y diseño sísmico de edificios en México - Cambios necesarios". Revista de Ingeniería Sísmica, No.86, pp. 89-118, México D.F., México.

Salinas, R., Rodríguez, M.E. y Sánchez, R. (2011), "Evaluación de procedimientos de diseño sísmico en México con base en resultados de ensayes de una estructura en mesa vibradora", XVIII Congreso Nacional de Ingeniería Sísmica. Aguascalientes, México.

Salinas, R. (2013), "Evaluación del desempeño sísmico de sistemas estructurales innovadores", Tesis de Doctorado, Facultad de Ingeniería, Universidad Nacional Autónoma de México. México.

Salinas, R. y Rodríguez, M.E. (2013), “Diseño sísmico de sistemas muro-marco con muros estructurales 
autocentrados", Artículo (en preparación) para posible publicación en la Revista de Ingeniería Sísmica.

Sánchez, R. (2008), "Diseño sísmico de edificios con sistemas duales regulares", Tesis de Doctorado, Facultad de Ingeniería, Universidad Nacional Autónoma de México. México.

Stone, W.C., Cheok, G. S., y Stanton, J. F., (1995), "Performance of hybrid moment-resisting precast beam-column concrete connections subjected to cyclic loading", ACI Structural Journal, V. 92, No 2, pp. 229-249.

Uang, C. (1991), "Establishing R (or Rw) and Cd Factors for Building Seismic Provisions", Journal of Structural Engineering ASCE, Vol. 117, No 1, pp.19-28.

Wallace, J. (1989), "BIAX, User manual - A computer program for the analysis of reinforced concrete sections", Universidad de California en Berkeley, CA, EE.UU. 